\title{
Linker modulated peroxide electrosynthesis using metal-organic nanosheets
}

\author{
Kiran Kuruvinashetti ${ }^{1}$, Nikolay Kornienko ${ }^{1 *}$ \\ ${ }^{1}$ Department of Chemistry, University of Montreal, 1375 Avenue Thérèse-Lavoie-Roux \\ Montreal, QC H2V 0B3, Canada \\ *Corresponding author: nikolay.kornienko@umontreal.ca
}

\begin{abstract}
:
The electrochemical synthesis of hydrogen peroxide $\left(\mathrm{H}_{2} \mathrm{O}_{2}\right)$, a widely used oxidant, is emerging as a green alternative to the conventional anthraquinone method. In this work, Ni-based metalorganic nanosheet (Ni-MONs) catalysts constructed using a variety of linkers were studied as oxygen reduction catalysts. Using a host of analytical techniques, we reveal how modulating the terephthalic acid linker with hydroxy, amine, and fluorine groups impacts the resulting physical and electronic structure of the $\mathrm{Ni}$ catalytic sites. These changes further impact the selectivity for $\mathrm{H}_{2} \mathrm{O}_{2}$, with the Ni-Amine-MON reaching near $100 \%$ Faradaic efficiency at minimal overpotential for the $2 e^{-} \mathrm{H}_{2} \mathrm{O}_{2}$ pathway in alkaline electrolyte. Finally, we translate the NiAmine-MON catalyst to a gas-diffusion reaction geometry and demonstrate a $\mathrm{H}_{2} \mathrm{O}_{2}$ partial current density of $200 \mathrm{~mA} / \mathrm{cm}^{2}$ while maintaining $85 \%$ Faradaic efficiency. In all, this study puts forth a simple route to catalyst modulation for highly effective $\mathrm{H}_{2} \mathrm{O}_{2}$ electrosynthesis.
\end{abstract}




\section{Introduction:}

Hydrogen peroxide $\left(\mathrm{H}_{2} \mathrm{O}_{2}\right)$ is an environmentally benign oxidant with diverse industrial applications, including paper-bleaching, waste-water treatment and chemical manufacturing. While conventionally produced via the anthraquinone process, there is a growing push to generate $\mathrm{H}_{2} \mathrm{O}_{2}$ through electrochemical means. ${ }^{[1]}$ Doing so would circumvent the necessity of using $\mathrm{H}_{2}$ and volatile $\mathrm{H}_{2} / \mathrm{O}_{2}$ mixtures, as well as issues with catalyst recyclability. Further, electrochemical $\mathrm{H}_{2} \mathrm{O}_{2}$ synthesis would enable a decentralization of its production, given the relative simplicity of the equipment necessary for this. Typically, $\mathrm{H}_{2} \mathrm{O}_{2}\left(\right.$ or $\mathrm{HO}_{2}{ }^{-}$in basic solutions) is produced via the $2 e^{-}$reduction of $\mathrm{O}_{2}$, a process that is in competition with the thermodynamically easier $4 e^{-}$reduction pathway to $\mathrm{H}_{2} \mathrm{O}$. A continual challenge in this direction is to develop both an understanding of the reaction process and factors that dictate $\mathrm{H}_{2} \mathrm{O}_{2}$ selectivity, as well as the continual development of effective catalytic systems.

In general noble metals, ${ }^{[2]}$ transition metal-based materials, ${ }^{[3]}$ and carbon nanostructures ${ }^{[4]}$ have emerged as promising $\mathrm{H}_{2} \mathrm{O}_{2}$ selective catalysts with each possessing their advantages and drawbacks. One recurrent theme is the importance of identifying of the most active catalytic motifs within catalysts that possess a wide variety of surface terminations and possible active sites. An emerging class of materials beginning to find unique uses in electrochemical systems is organic/inorganic hybris systems such as metal-organic frameworks (MOFs), covalent-organic frameworks (COFs) and their derivatives. ${ }^{[5]}$ A particular advantage that they hold is their molecularly well-defined catalytic sites and modular structure that could enable the extraction of discreet structure-activity relationships. As such, only few metal-organic structures have been applied for $\mathrm{H}_{2} \mathrm{O}_{2}$ synthesis. ${ }^{[6]}$ In this work, we build on these previous studies and study the effects of linker identity on Ni-based metal-organic nanosheets (MONs). We construct MONs using terephthalic acid and several derivatives and characterize the resulting materials' physical and electronic structure with an array of techniques (Fig. 1). We show how subtle differences in the Ni electronic structure conferred through changes in linker identity led to significant changes in $\mathrm{H}_{2} \mathrm{O}_{2}$ selectivity, with the Ni-Amine-MON exhibiting near 100\% Faradaic efficiency (FE) towards $\mathrm{H}_{2} \mathrm{O}_{2}$. Finally, we translate the catalyst from a conventional 3-electrode setup to a gasdiffusion electrode (GDE) - based reactor and attain industrially-relevant $\mathrm{H}_{2} \mathrm{O}_{2}$ partial current densities of $200 \mathrm{~mA} / \mathrm{cm}^{2}$ while keeping an $85 \% \mathrm{FE}$ for $\mathrm{H}_{2} \mathrm{O}_{2}$.

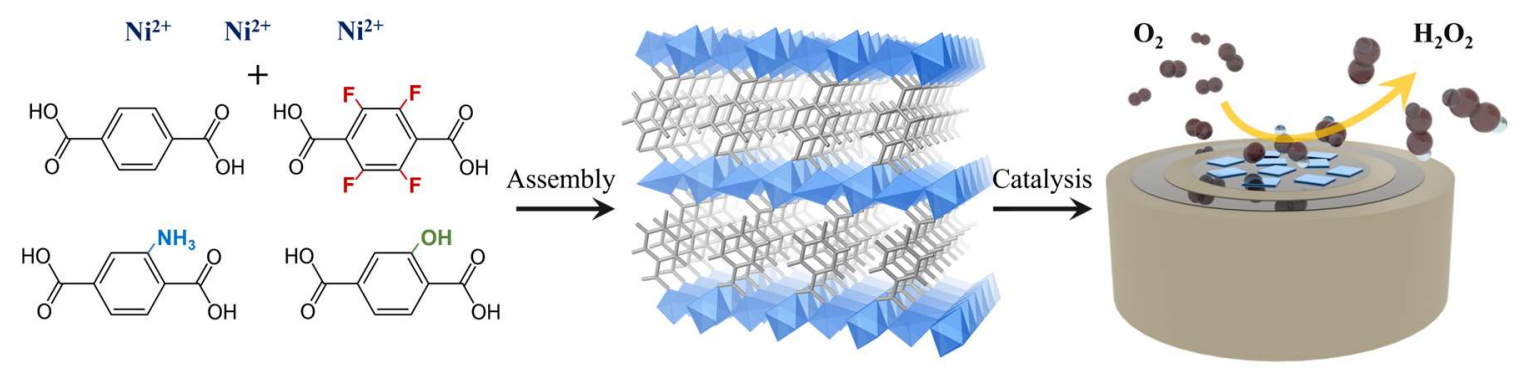

Figure 1: MON assembly from terephthalic acid linkers appended with various functional groups. The effects of the linkers on the MON catalytic properties, in particular their selectivity for $\mathrm{H}_{2} \mathrm{O}_{2}$ production from $\mathrm{O}_{2}$, were then characterized. 


\section{Results and Discussion}

All MONs were synthesized using an adaptation of previously established methods. ${ }^{[7]}$ Briefly, $\mathrm{Ni}^{2+}$ was sonicated together with terephthalic acid or its derivatives to assemble layered structures containing sheets $\mathrm{NiO}_{6}$ octahedra. Four types of Ni-MONs were synthesized and named in accordance with the type of linker used (terephthalic acid - Ni-MON; tetrafluoroterephthalic acid - Ni-F-MON; hydroxyterephthalic acid - Ni-OH-MON, aminoterephthalic acid - Ni-Amine-MON). The aim here was to have the electron donating or withdrawing groups on the linker modulate the electronic structure and consequently, catalytic performance of the Ni active sites for the oxygen reduction reaction. Following synthesis and purification, the materials were characterized with a host of techniques. First infrared spectroscopy showed revealed strong bands around 1600-1700 (C=O stretch) for all MONs, revealing the presence of terephthalate (Fig. 2a). Next, X-ray diffraction (XRD) was employed to characterize the crystallinity of the MONs (Fig. 2b). While the Ni-MON and Ni-Amine-MON assembled into the expected phase [no. 985792, Cambridge Crystallographic Data Centre], ${ }^{[7]}$ the $\mathrm{Ni}-\mathrm{OH}-\mathrm{MON}$ remained largely amorphous as its smaller grain size did not lead to strong diffraction peaks. However, electron diffraction measurements did indicate a weak degree of crystallinity that could be observed (Fig. S10). Further, the Ni-F-MON assembled into a layereddouble hydroxide (LDH) structure. However, as the F-component was detected throughout the material via elemental mapping through transmission electron microscopy (TEM) measurements (Fig. S9) we believe that the linkers were confined to the outer portion of the MON instead. Atomic force microscopy (AFM) was then used to provide another layer of structural information (Fig. 2c and S3-7). The measurements reveal all of the materials to possess a flakelike morphology and thicknesses on the order of $10 \mathrm{~nm}$. TEM images (Fig. 2d and S8-11) further show that the MONs featured a rough morphology. Finally, x-ray photoelectron spectroscopy (XPS) was utilized to measure the electronic structure of the Ni components. The $\mathrm{Ni} 2 \mathrm{p}_{3 / 2}$ peaks for all MONs are shown in Fig. 2e and slight shifts in their binding energy roughly match the electron donating/withdrawing nature of the functional groups on the terephthalate ligands, with a $0.31 \mathrm{eV}$ separation between the peaks of the Ni-F-MON and Ni-Amine-MON. 
a

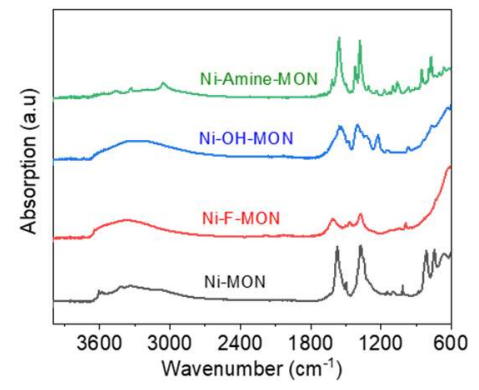

C

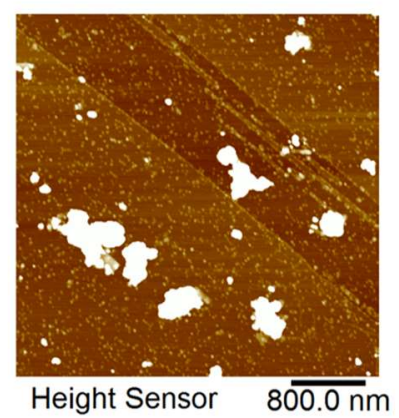

b

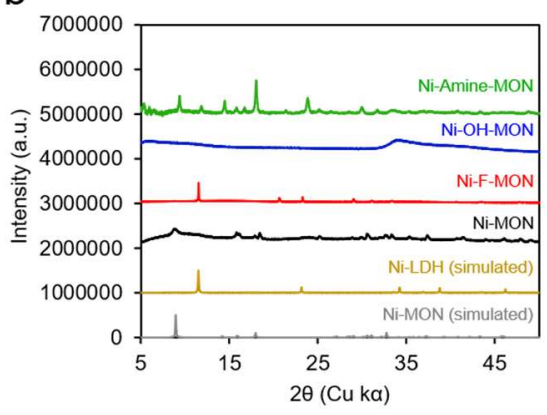

d

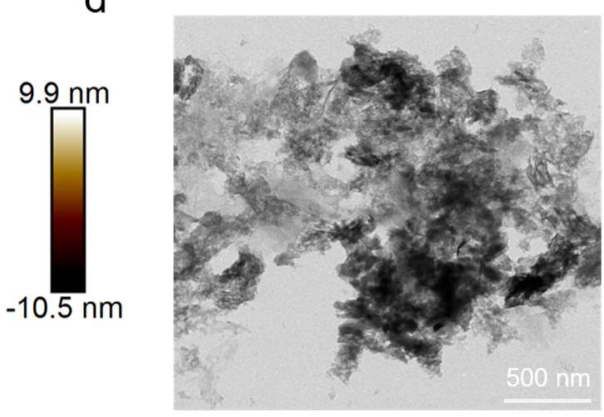

e

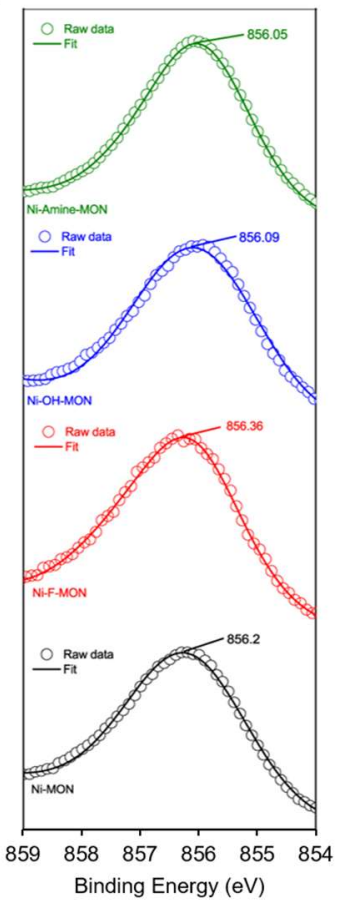

Figure 2: Characterization of the synthesized Ni-MONs. IR measurements show the presence of the terephthalate within the materials (a) while XRD measurements (b) elucidate the resultant crystal structure. AFM measurements (c) illustrate the flake-like morphology as TEM measurements (d) point to the roughness of the materials. Finally, XPS spectra (e) measure the electronic structure of the Ni-MONs and differences conferred by the ligands.

\section{Electrochemical studies:}

The electrochemical performance of Ni-based MONs towards peroxide synthesis was next studied with rotating ring disk electrode (RRDE) system in aqueous $1 \mathrm{M} \mathrm{KOH}$ electrolyte. To do so, a catalyst in was first prepared form the dry MONs in an ethanol/water/nafion/carbon nanotube (CNT) dispersion, which was subsequently drop cast onto the working electrode. In an $\mathrm{O}_{2}$-saturated electrolyte, each MON featured a current onset around $0.8 \mathrm{~V}_{\mathrm{RHE}}$ (Fig. 3a). However, the ring, used to oxidize peroxide $\left(\mathrm{HO}_{2}{ }^{-}\right.$in alkaline electrolyte), revealed stark differences between their selectivity. The Ni-MON and Ni-F-MON exhibited rather low peroxide selectivity on the order of $45-70 \%$ (Fig. 3b). In contrast, the Ni-Amine-MON featured selectivity above $90 \%$ from $0.45 \mathrm{~V}_{\mathrm{RHE}}$. The mass loading of the MONs $\left(0.2 \mathrm{mg} / \mathrm{cm}^{2}\right)$ was identical for all catalysts and the quantity of electrochemically accessible Ni sites (Fig. S14) was similar so changes in peroxide selectivity are attributed to intrinsic differences between the catalysts. This is also reflected in their differing Tafel slopes (Fig. S15), in which the higher peroxide selectivity is correlated with higher Tafel slope values. While we do could not extensively characterize the MONs after electrochemical experiments on an RRDE, we measured their XRD patterns after prolonged electrolysis on a carbon paper electrode instead (Fig. S17). The results indicate that the MONs unexpectedly lose their long-range crystallinity during the process and only very 
weak peaks attributed to a residual LDH phase remain. Likely, during the RRDE measurements, the MONs likely lose their long-range order as well, though retain the local coordination that gives rise to their differing performance.
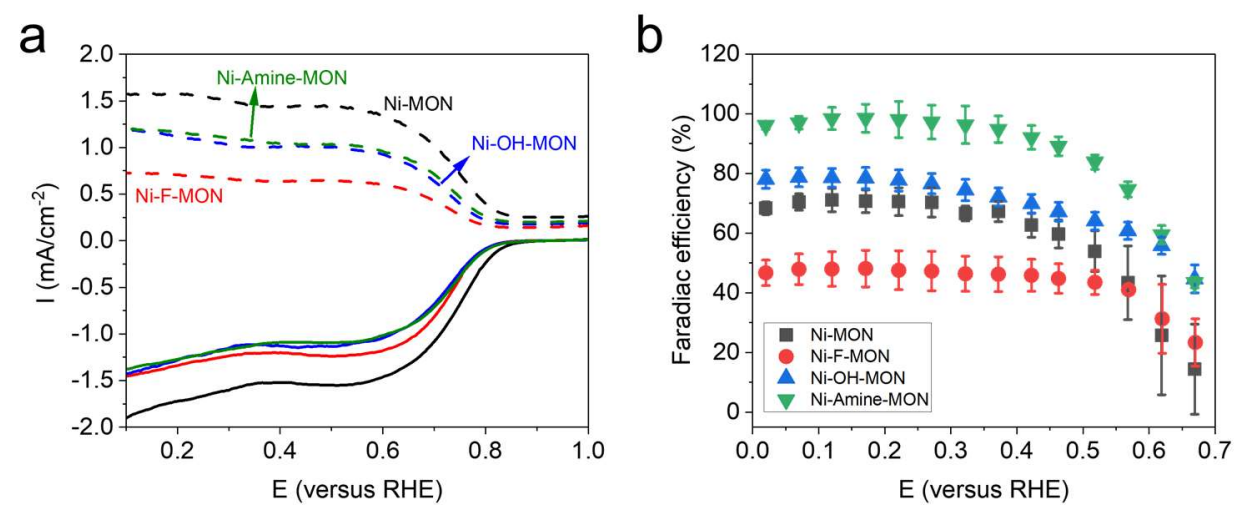

Figure 3: Electrochemical measurements. Linear sweep voltammetry (LSV) measurements of Ni-MONs (a), and Faradaic efficiency for peroxide calculated from at least three independent measurements (b).

To translate our initial catalytic results to a practical peroxide electrosynthesis system, we moved to use a gas-diffusion electrode setup (Fig. 4a). To do so, we deposited the same catalyst ink on a gas diffusion electrode, featuring a gas-permeable support and surface, which enabled us to circumvent the low solubility of $\mathrm{O}_{2}$ in aqueous solutions. As the Ni-Amine-MON featured the highest selectivity for peroxide in RRDE measurements, this was our chosen material for further analysis. The peroxide produced was quantified through a simple $\mathrm{Ce}^{4+}$-based colorimetric assay after prolonged (approx. $30 \mathrm{~min}$ ) electrolysis times (Fig. S18). The partial current density attributed to peroxide production started to slowly increase at $0.72 \mathrm{~V}_{\mathrm{RHE}}$ and reached an impressive $\sim 200 \mathrm{~mA} / \mathrm{cm}^{2}$ at $0.52 \mathrm{~V}_{\mathrm{RHE}}$ (Fig. $4 \mathrm{~b}$ ), after which it began to slightly drop as the $4 e^{-}$ oxygen reduction pathway began to contribute. At this high partial current density, the FE for peroxide $85 \%$ (Fig. 4c). These metrics are comparable to the highest performing state-of-the-art systems recently published. ${ }^{[8]}$ Further, the system could continually produce peroxide for upwards of 75 hours (Fig. S19). 


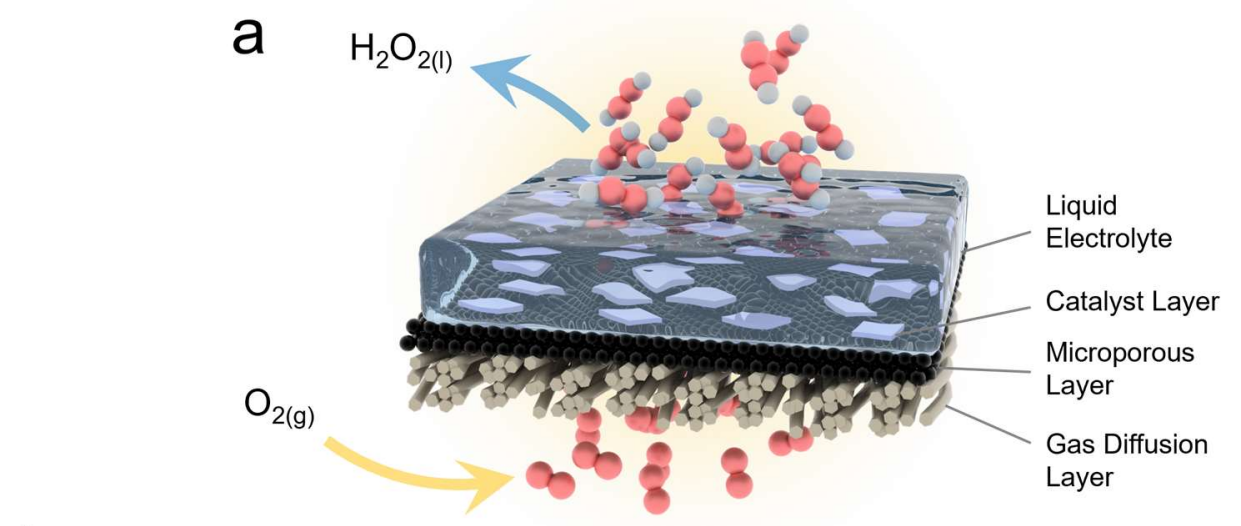

b

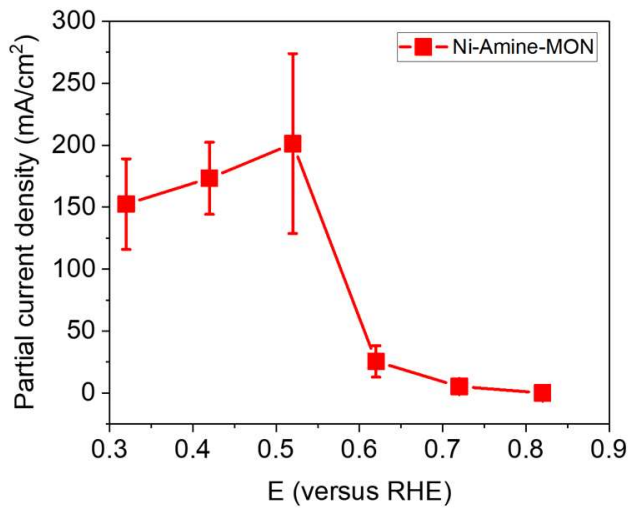

C

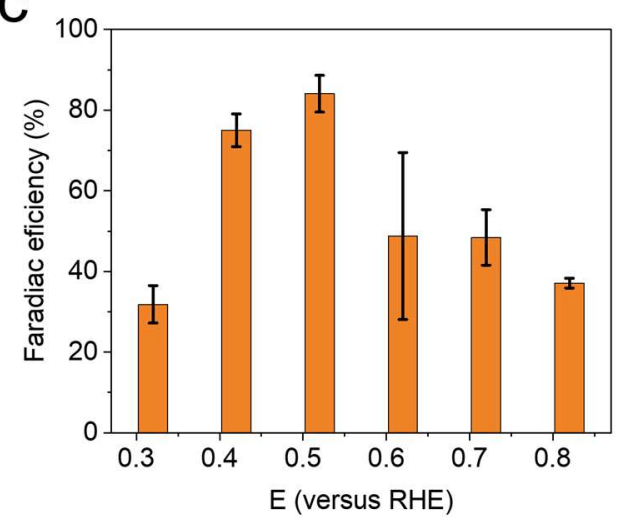

Figure 4: Illustration of gas diffusion electrode set up for peroxide synthesis (a). With the $\mathrm{Ni}$ Amine-MON in this reactor geometry, we recorded a partial current density of up to $200 \mathrm{~mA} / \mathrm{cm}^{2}$ (b) at a FE of $85 \%$ (c).

\section{Concluding remarks:}

In all, this work exemplified how a modular approach could be exploited to generate highly effective catalytic systems. In particular, by using an amine-appended terephthalic acid linker to assemble Ni-based MONs, we have enhanced the catalytic activity of the resultant material to attain near $100 \%$ selectivity for the $2 e^{-}$oxygen reduction reaction pathway to peroxide. Further, translating this catalyst onto a GDE-based reactor, partial current densities of $200 \mathrm{~mA} / \mathrm{cm}^{2}$ were reached while maintaining a high $85 \% \mathrm{FE}$. While there are still open questions regarding the exact identity of the active sites (e.g. defects, local coordination), the nature of the MON structural dynamics during catalysis, and the precise reaction mechanism on the MON surfaces, this study opens up promising avenues to pursue in the design and understanding of peroxideproducing electrosynthetic systems. 
Acknowledgements: The authors acknowledge ACS PRF New Directions Grant 65093-ND5.

\section{Reference:}

[1] aS. Siahrostami, S. J. Villegas, A. H. Bagherzadeh Mostaghimi, S. Back, A. B. Farimani, H. Wang, K. A. Persson, J. Montoya, ACS Catal. 2020, 10, 7495-7511; bS. C. Perry, D. Pangotra, L. Vieira, L.-I. Csepei, V. Sieber, L. Wang, C. Ponce de León, F. C. Walsh, Nat. Rev. Chem. 2019, 3, 442-458; cY. Jiang, P. Ni, C. Chen, Y. Lu, P. Yang, B. Kong, A. Fisher, X. Wang, Adv. Energy Mater. 2018, 8, 1801909.

[2] aJ. S. Jirkovský, I. Panas, E. Ahlberg, M. Halasa, S. Romani, D. J. Schiffrin, J. Am. Chem. Soc. 2011, 133, 19432-19441; bC. H. Choi, M. Kim, H. C. Kwon, S. J. Cho, S. Yun, H.-T. Kim, K. J. J. Mayrhofer, H. Kim, M. Choi, Nat. Commun. 2016, 7, 10922; cC. Yang, S. Bai, Z. Yu, Y. Feng, B. Huang, Q. Lu, T. Wu, M. Sun, T. Zhu, C. Cheng, L. Zhang, Q. Shao, X. Huang, Nano Energy 2021, 89, 106480.

[3] aH. Shen, L. Pan, T. Thomas, J. Wang, X. Guo, Y. Zhu, K. Luo, S. Du, H. Guo, G. J. Hutchings, J. P. Attfield, M. Yang, Cell Rep. Phys. Sci. 2020, 1, 100255; bQ. Zhang, X. Tan, N. M. Bedford, Z. Han, L. Thomsen, S. Smith, R. Amal, X. Lu, Nat. Commun. 2020, 11, 4181; CP. T. Smith, Y. Kim, B. P. Benke, K. Kim, C. J. Chang, Angew. Chem. Int. Ed. 2020, 59, 4902-4907.

[4] aY. Xia, X. Zhao, C. Xia, Z.-Y. Wu, P. Zhu, J. Y. Kim, X. Bai, G. Gao, Y. Hu, J. Zhong, Y. Liu, H. Wang, Nat. Commun. 2021, 12, 4225; bH. W. Kim, M. B. Ross, N. Kornienko, L. Zhang, J. Guo, P. Yang, B. D. McCloskey, Nat. Catal. 2018, 1, 282-290; cZ. Lu, G. Chen, S. Siahrostami, Z. Chen, K. Liu, J. Xie, L. Liao, T. Wu, D. Lin, Y. Liu, T. F. Jaramillo, J. K. Nørskov, Y. Cui, Nat. Catal. 2018, 1, 156-162; dZ. Wang, Q.-K. Li, C. Zhang, Z. Cheng, W. Chen, E. A. McHugh, R. A. Carter, B. I. Yakobson, J. M. Tour, ACS Catal. 2021, 11, 2454-2459.

[5] aH. Wang, Q.-L. Zhu, R. Zou, Q. Xu, Chem 2017, 2, 52-80; bB. Wu Hao, W. Lou Xiong, Sci. Adv., 3, eaap9252.

[6] aE. M. Miner, T. Fukushima, D. Sheberla, L. Sun, Y. Surendranath, M. Dincă, Nat. Commun. 2016, 7, 10942; bM. Wang, X. Dong, Z. Meng, Z. Hu, Y.-G. Lin, C.-K. Peng, H. Wang, C.-W. Pao, S. Ding, Y. Li, Q. Shao, X. Huang, Angew. Chem. Int. Ed. 2021, 60, 11190-11195; cM. Wang, N. Zhang, Y. Feng, Z. Hu, Q. Shao, X. Huang, Angew. Chem. Int. Ed. 2020, 59, 14373-14377.

[7] S. Zhao, Y. Wang, J. Dong, C.-T. He, H. Yin, P. An, K. Zhao, X. Zhang, C. Gao, L. Zhang, J. Lv, J. Wang, J. Zhang, A. M. Khattak, N. A. Khan, Z. Wei, J. Zhang, S. Liu, H. Zhao, Z. Tang, Nat. Energy 2016, 1, 16184.

[8] aQ. Zhang, M. Zhou, G. Ren, Y. Li, Y. Li, X. Du, Nat. Commun. 2020, 11, 1731; bC. Xia, Y. Xia, P. Zhu, L. Fan, H. Wang, Science 2019, 366, 226-231; cB. Garza-Campos, D. Morales-Acosta, A. Hernández-Ramírez, J. L. Guzmán-Mar, L. Hinojosa-Reyes, J. Manríquez, E. J. Ruiz-Ruiz, Electrochim. Acta 2018, 269, 232-240. 
Supplementary information for:

Linker modulated peroxide electrosynthesis using metal-organic nanosheets

Kiran Kuruvinashetti ${ }^{1 *}$, Nikolay Kornienko ${ }^{1}$

${ }^{1}$ Department of Chemistry, University of Montreal, 1375 Avenue Thérèse-Lavoie-Roux

Montreal, QC H2V 0B3, Canada

Corresponding author: nikolay.kornienko@umontreal.ca

\section{Materials and Methods}

Chemicals: $\mathrm{NiCl}_{2} 6 \mathrm{H}_{2} \mathrm{O}$ (99.99\% AR grade) purchased from Sigma Aldrich. Terephthalic acid , Tetrafluoroterephthalic acid, hydroxy-terephthalic acid, Aminoterepthalic acid, Nafion solution ((5 wt \%), $1 \mathrm{M} \mathrm{KOH} \mathrm{(electrolyte),} \mathrm{Triethylamine} \mathrm{(TEA),} \mathrm{N-N} \mathrm{dimethylformamide} \mathrm{(DMF),}$ Deionized water $(18 \mathrm{M} \Omega)$ were used in all experimentations.

Synthesis of Ni-metal organic nanosheets (MON): The metal organic nanosheets were synthesized using: DMF $16 \mathrm{ml}$, ethanol $1 \mathrm{ml}$, DI water $1 \mathrm{ml}$, all mixed together. $0.75 \mathrm{mmol}$ terephthalic acid was added to the solution and ultrasonicated for 10 minutes. Further $0.75 \mathrm{mmol}$ $\mathrm{NiCl}_{2} 6 \mathrm{H}_{2} \mathrm{O}$ were added. When the $\mathrm{Ni}^{2+}$ salts dissolved in the solution $0.4 \mathrm{ml}$ TEA was added to it. Further the solution was stirred for 10 minutes to obtain a uniform colloidal dispersion. The prepared colloidal solution was ultrasonicated for 8 hours at $(40 \mathrm{kHz})$ under airtight conditions. After 8 hours, the solution was centrifuged and washed with ethanol for 4 to 5 times and the obtained Ni-MON was dried in vacuum oven at $85^{\circ} \mathrm{C}$ in air.

Synthesis of Ni-F-MON: The preparation process of Ni-F-MON is same as that of the Ni-MON, However, the only difference is instead of terephthalic acid, $0.75 \mathrm{mmol}$ Tetrafluoroterephthalic acid was added. The final product obtained Ni-F-MON was washed with ethanol for 4 to 5 times and the obtained Ni-F-MON was dried in vacuum oven at $85^{\circ} \mathrm{C}$ in air.

Synthesis of Ni-OH-MON: The preparation process of Ni-OH-MON is same as that of the $\mathrm{Ni}-$ MON, but the only difference is instead of Terephthalic acid, $0.75 \mathrm{mmol}$ hydroxy-terephthalic acid was added. The final product obtained Ni-OH-MON was washed with ethanol for 4 to 5 times and the obtained Ni-F-MON was dried in vacuum oven at $85^{\circ} \mathrm{C}$ in air.

Synthesis of Ni-Amine-MON: The preparation process of Ni-Amine-MON is same as that of the Ni-MON, but the only difference is instead of Terephthalic acid, $0.75 \mathrm{mmol}$ amino terephthalic acid is added. The final product obtained Ni-Amine-MON was washed with ethanol for 4 to 5 times and the obtained Ni-F-MON was dried in vacuum oven at $85^{\circ} \mathrm{C}$ in air. 
Preparation of catalytic ink for working electrode: $0.2 \mathrm{ml}$ DI water, $0.3 \mathrm{ml}$ ethanol, and 0.02 $\mathrm{ml}$ Nafion (5\% solution) and $2.2 \mathrm{mg}$ of catalyst (Ni-MON, Ni-F-MON, Ni-OH-MON, NiAmine-MON), 5\% (w/v) carbon nanotubes $(\mathrm{CNT})$ (for enhanced conductivity) ultrasonicated for 2 hours.

Electrochemical characterization (LSV): The electrochemical tests (LSV) were performed using Palmsense bi-potentiostat three electrode system. $\mathrm{Ag} / \mathrm{AgCl}$ electrodes were served as reference electrodes. The $\mathrm{Ag} / \mathrm{AgCl}$ was periodically referenced to a master electrode to ensure that the potential did not significantly drift. Carbon rods were employed as counter electrode. Then, $10 \mu \mathrm{l}$ of catalytic ink on the glassy carbon electrode $\left(0.2 \mathrm{~cm}^{2}\right)$ working electrode. Oxygen saturated $1 \mathrm{M}$ potassium hydroxide $(\mathrm{KOH})$ was used as electrolyte. The activity and selectivity were studied by linear sweep voltammetry (LSV). The LSV's were performed at scan rate of 10 $\mathrm{mV} / \mathrm{s}$ at $1600 \mathrm{rpm}$.

Selectivity calculation: Peroxide selectivity of the MON's on the surface of ring and disk electrode was calculated based on the current of both disc and ring electrode [19] using the equation

FE was calculated by: $F E(\%)=\frac{\frac{I_{\text {ring }}}{N}}{I_{\text {disk }}} \times 100$

Where $\mathrm{N}$ - collection efficiency; 0.28 for the RRDE system

A Bio-Logic SP-200 Potentiostat was used for measurements using a GDE electrode with an ohmic drop compensation set to $85 \%$. This was performed prior to electrolysis measurements by recording the impedance at open circuit at $100 \mathrm{KHz}$.

Electrochemical characterization (CV): The electrochemical tests (CV) were performed using Bio-Logic SP-200 Potentiostat set up in a three-electrode system. The catalyst $1 \mathrm{mg} / \mathrm{cm}^{2}$ drop casted on carbon paper were served as working electrode. $\mathrm{Ag} / \mathrm{AgCl}$ as reference electrode and carbon rods as counter electrode. Oxygen saturated $1 \mathrm{M} \mathrm{KOH}$ was used as electrolyte.

Characterization by TEM and EDS spectra: TEM analysis and EDS spectra were performed in the McGill University. $0.3 \mathrm{ml}$ ethanol, $0.2 \mathrm{ml}$ DI water, 0.05 nafion and $2.2 \mathrm{mg}$ of catalyst MON's were sonicated for 2 hours and diluted (1: 10000) in Di water. The diluted catalyst solution of approximately $1 \mu \mathrm{l}$ was deposited on copper grid supporting a thin electron transparent carbon film. And the TEM images were comprehended.

Characterization by XPS: To understand the electronic structure of the catalyst X -ray photoelectron spectroscopy was used. The high-resolution X-ray photoelectron spectroscopy (XPS) analysis of the catalysts was performed using a Vacuum Generated Escalab 220i-XL system in the Institute National de la Recherche Scientifique Centre Energie Materiaux et communications. Mg was used as an X-ray source. 
Characterization by XRD: The crystalline structures of catalysts MON's were identified by powder X-ray diffraction (XRD, Bruker D8 Advance diffractometer) using $\mathrm{Cu}-\mathrm{K} \alpha$ radiation source $(\lambda 1.5418 \AA)$ in the University of Montreal.

Characterization by Infrared spectroscopy: IR spectra were acquired on a ThermoFischer Nicolet 380 FTIR. 400 scans were acquired for each measurement at a resolution of $4 \mathrm{~cm}^{-1}$ ).

Characterization by AFM: Average height and diameter of the catalyst MON's were performed using the AFM Brucker. The dry Ni-based samples was dispersed in the ethanol and water and nafion solution to have better dispersity and the sample was sonicated for 1.5 to 2 hours. The sonicated samples were drop casted on the surface of mica sheets to perform the AFM.

Characterization by DLS: Average diameter of the catalyst MON's was measured by the dynamic light scattering (DLS). The $0.3 \mathrm{ml}$ ethanol, $0.2 \mathrm{ml}$ DI water, 0.05 nafion and $2.2 \mathrm{mg}$ of catalyst MONs were sonicated for 2 hours and diluted (1: 1000) in DI water. The diluted samples were analyzed in cuvette to comprehend the average diameter of the catalysts.

Faradaic efficiency (FE) for the partial current density: FE was calculated by using the following formula:

$$
F E=\frac{\alpha n F}{Q}
$$

$\mathrm{FE}=\alpha n F / Q$

where $\alpha$ is electron transfer numbers, $n$ is the moles of the products, $F$ is the Faraday constant $\left(96485.3 \mathrm{C} \mathrm{mol}^{-1}\right), Q$ is the charge passed during the reaction.

Gas diffusion electrode set up: Three electrode set up was used. $\mathrm{Ag} / \mathrm{AgCl}$ was served as reference electrode, carbon rods were used as counter electrode, carbon cloth ( gas diffusion layer (GDL-CT (W1S1009, Fuel Cells Etc.) drop casted with the catalyst ink was employed as the working electrode. The Chronoamperometry data was recorded using Bio-Logic SP-200 Potentiostat (BioLogic Science Instruments, France).

Scaled up synthesis of peroxide: H-bridge reactor was used to synthesis of peroxide. Carbon cloth (GDL-CT (W1S1009, Fuel Cells Inc.) with an area $1.5 \mathrm{~cm}^{2}$ with catalyst ink (Ni-AmineMON) deposition of $4 \mathrm{mg} / \mathrm{cm}^{2}$ served as working electrode. $\mathrm{Ag} / \mathrm{AgCl}$ was employed as reference electrode and an isolated $\mathrm{Pt}$ wire within a fritted tube was served as the counter electrode.

$\mathrm{H}_{2} \mathrm{O}_{2}$ concentration measurement: The peroxide concentration was measured using titration of cerium sulfate $\mathrm{Ce}(\mathrm{SO})_{4}$. The yellow solution of the $\mathrm{Ce}^{4+}$ have been reduced by peroxide molecules to colorless solution. Based on this underlying principle, UV-vis spectroscopy was used to study to measure the concentration of $\mathrm{Ce}^{4+}$ before and after the reaction. The $399.5 \mathrm{~nm}$ wavelength's corresponding absorption intensity was noted. 
$3 \mathrm{mM} \mathrm{Ce}(\mathrm{SO})_{4}$ solution was prepared by dissolving $99.6 \mathrm{mg}$ of $\mathrm{Ce}\left(\mathrm{SO}_{4}\right)_{2}$ in $100 \mathrm{ml} 0.5 \mathrm{M}$ sulfuric acid solution. To get the calibration curve, the peroxide of known solution was added to $\mathrm{Ce}\left(\mathrm{SO}_{4}\right)_{2}$ solution and the measurements were performed using UV-Vis spectroscopy. Based on the intensity (y axis) and $\mathrm{Ce}^{4+}$ concentration the concentration of the peroxide was obtained

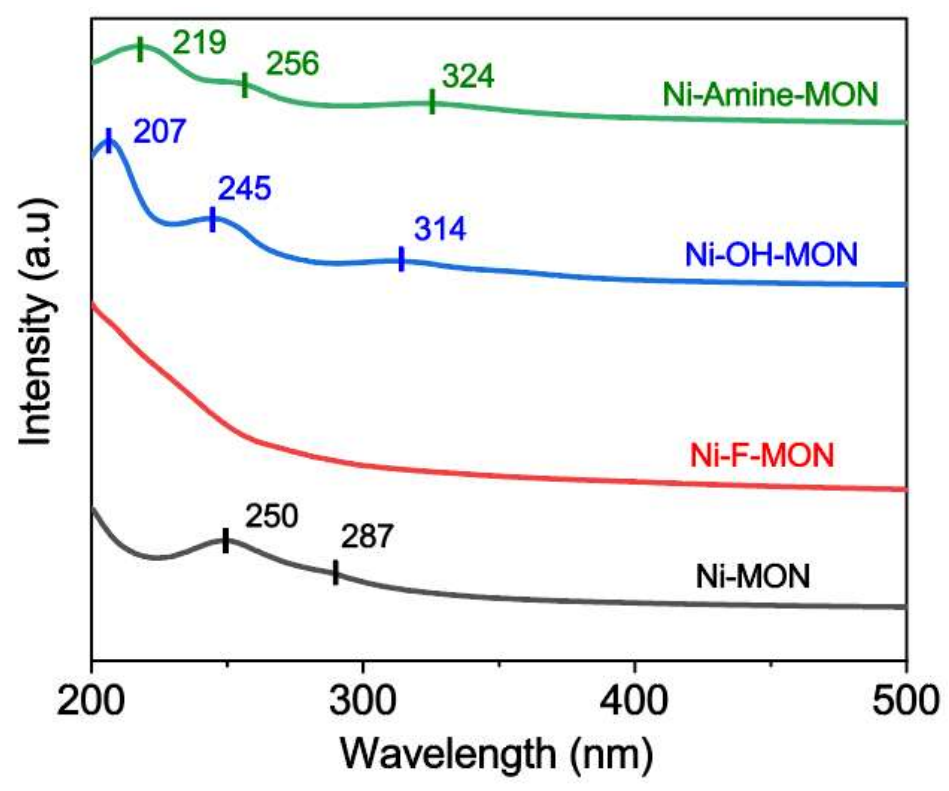

Figure S1: UV-Vis absorption spectroscopy of Ni-based MONs in DI water solution. 


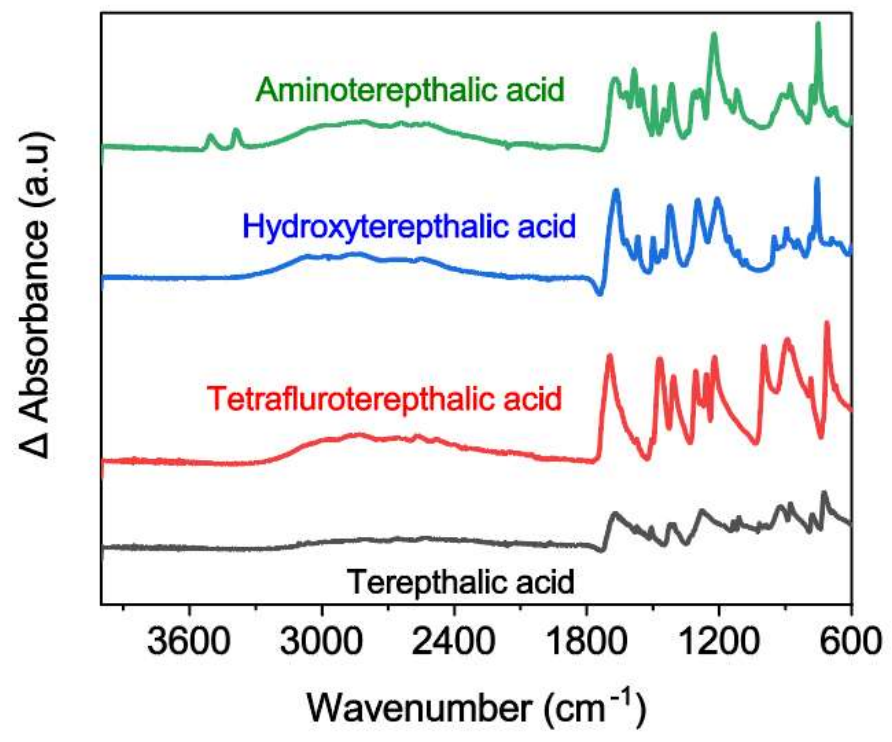

Figure S2: IR spectra of linkers of the corresponding Ni-based MONs. (Terephthalic acid - NiMON), (Tetrafluoroterephthalic acid - Ni-F-MON), (Hydroxyterephthalic acid - Ni-OH-MON) and Aminoterephthalic acid (Ni-Amine-MON). 
(a)

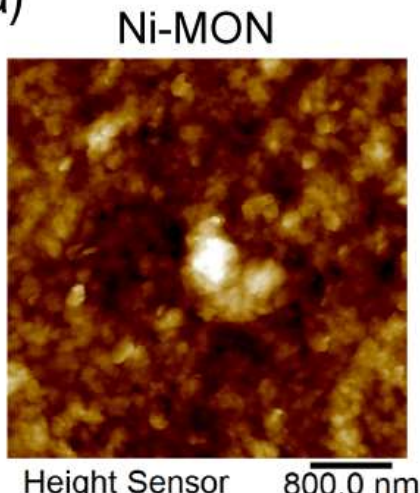

(c)
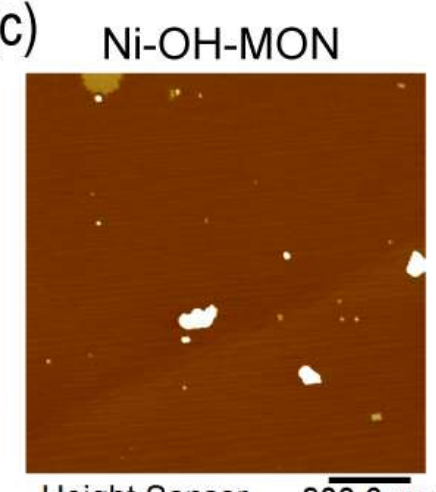

Height Sensor $8 \overline{800.0 \mathrm{n} m}$ (b)
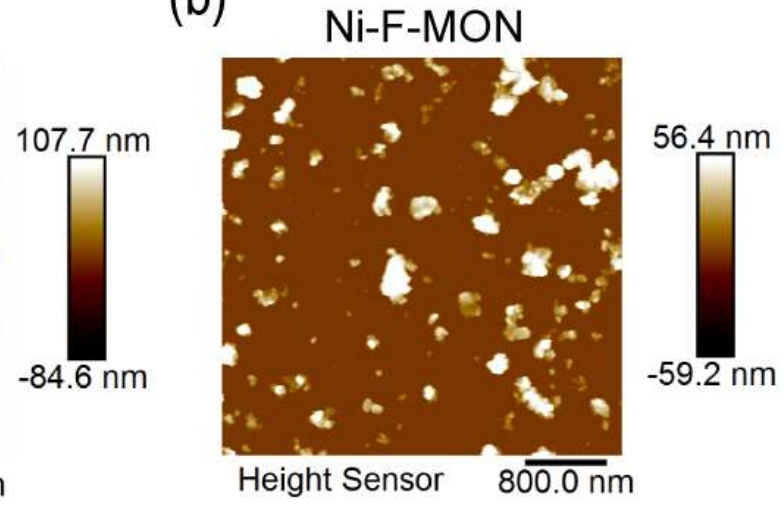

(d) Ni-Amine-MON
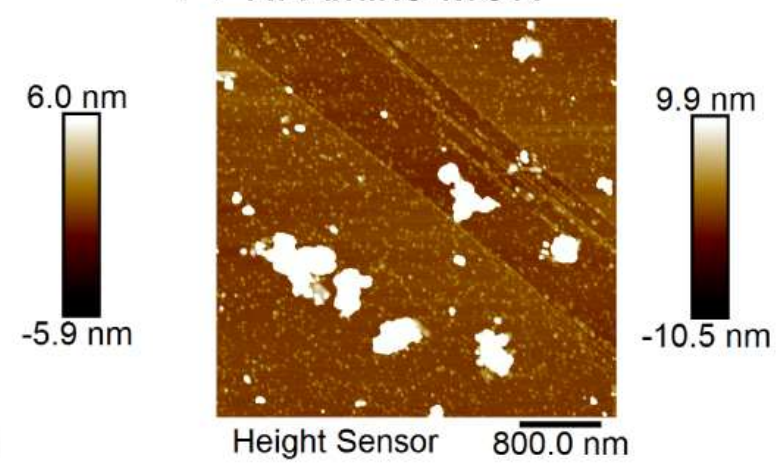

Figure S3. Atomic Force Microscopy (AFM) images of (a) Ni-MON (b) Ni-F- MON (c) Ni-OHMON (d) Ni-Amine-MON 

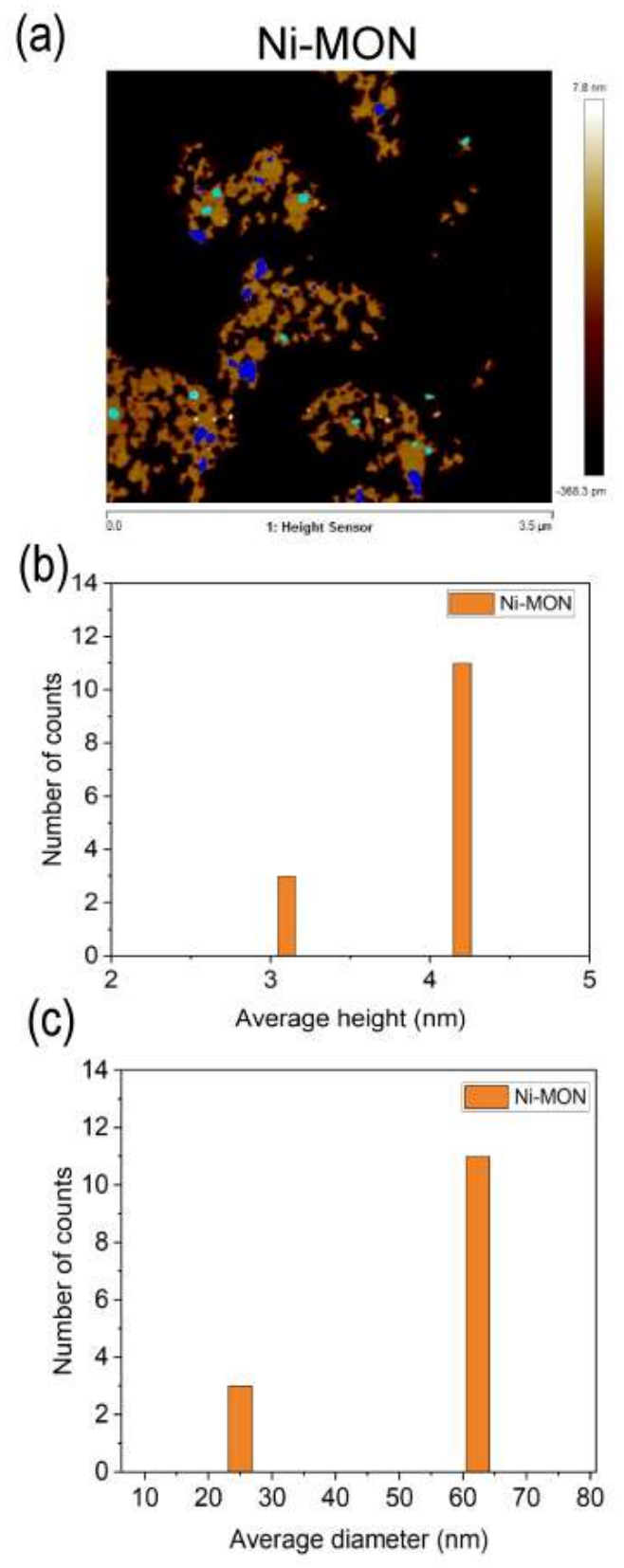

Figure S4. Average height and diameter of the Ni-MON characterized by AFM. (a) Imaging demonstrating the Ni-MON and the sample locations (shown with blue and cyan colors) at which their average height and diameter are analyzed (b) Average height of the Ni-MON samples; the heights of the Ni-MONs are around 3 to $4.5 \mathrm{~nm}$ and average diameter varies from $25 \mathrm{~nm}$ to 65 $\mathrm{nm}$. Y axis represents the number of counts at which the average values of height and diameters of Ni-MON are calculated. 


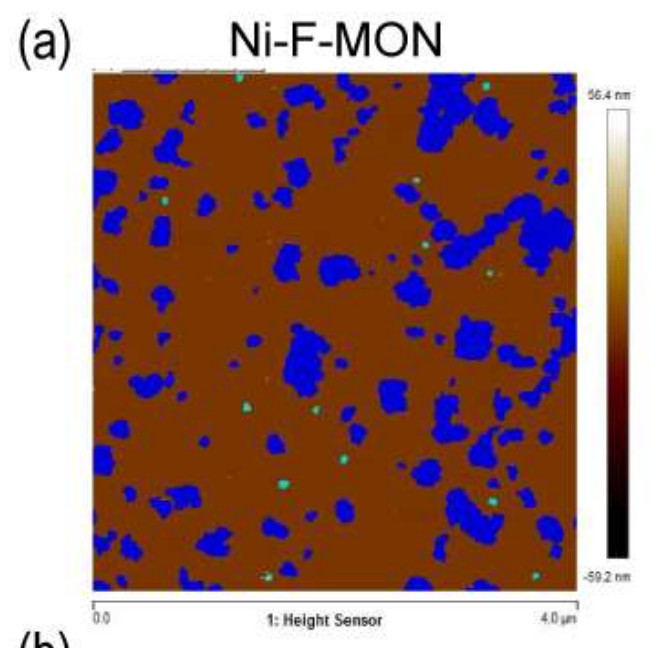

(b)

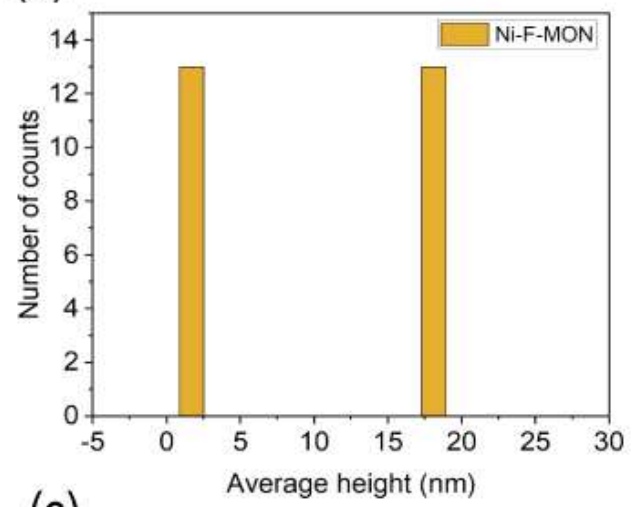

(c)

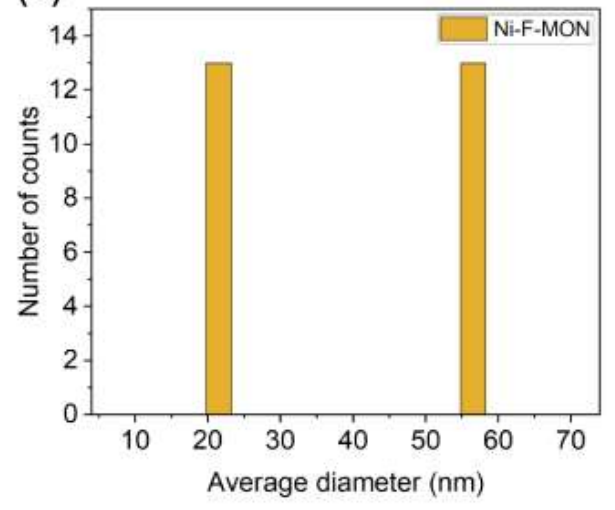

Figure S5. Average height and diameter of the Ni-F-MON characterized by AFM. (a) Imaging demonstrating the Ni-F-MON and the sample locations ( shown cyan colors) at which their average height and diameter are analyzed (b) Average height of the Ni-F-MON samples; the heights of the Ni-F-MONs are around 1 to $20 \mathrm{~nm}$ and average diameter varies from $20 \mathrm{~nm}$ to 60 $\mathrm{nm}$. Y axis represents the number of counts at which the average values of height and diameters of Ni-F-MON are calculated. 


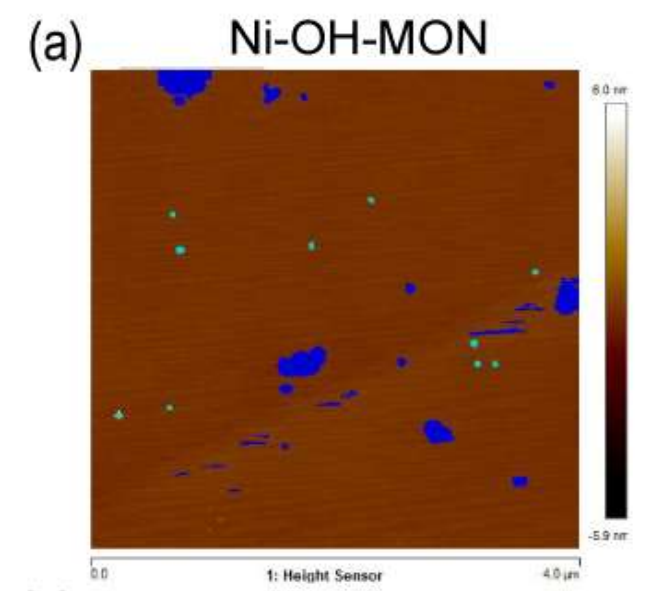

(b)

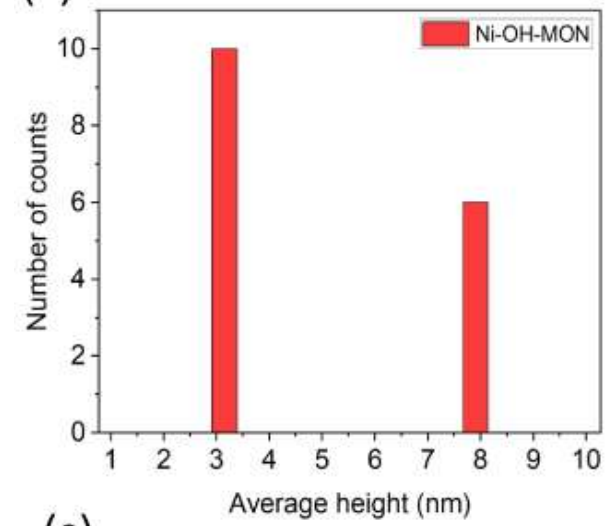

(c)

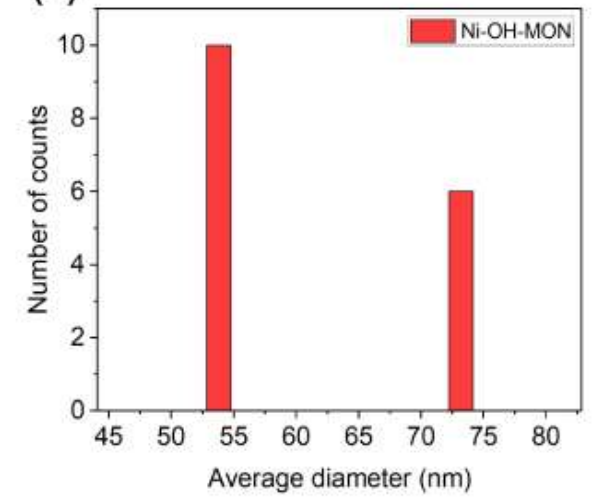

Figure S6. Average height and diameter of the Ni-OH-MON characterized by AFM. (a) Imaging demonstrating the Ni-OH-MON and the sample locations ( shown cyan colors) at which their average height and diameter are analyzed (b) Average height of the Ni-OH-MON samples; the heights of the Ni-OH-MONs are around 3 to $8 \mathrm{~nm}$ and average diameter varies from $50 \mathrm{~nm}$ to $75 \mathrm{~nm}$. Y axis represents the number of counts at which the average values of height and diameters of Ni-OH-MON are calculated. 


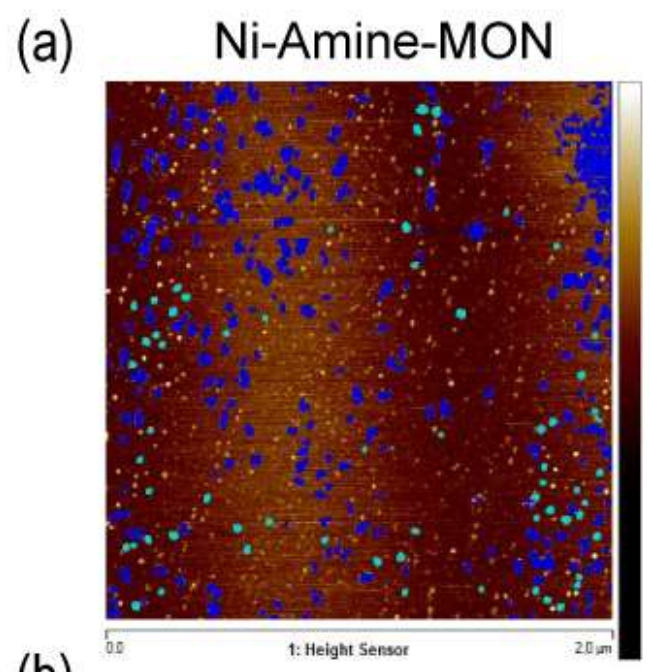

(b)
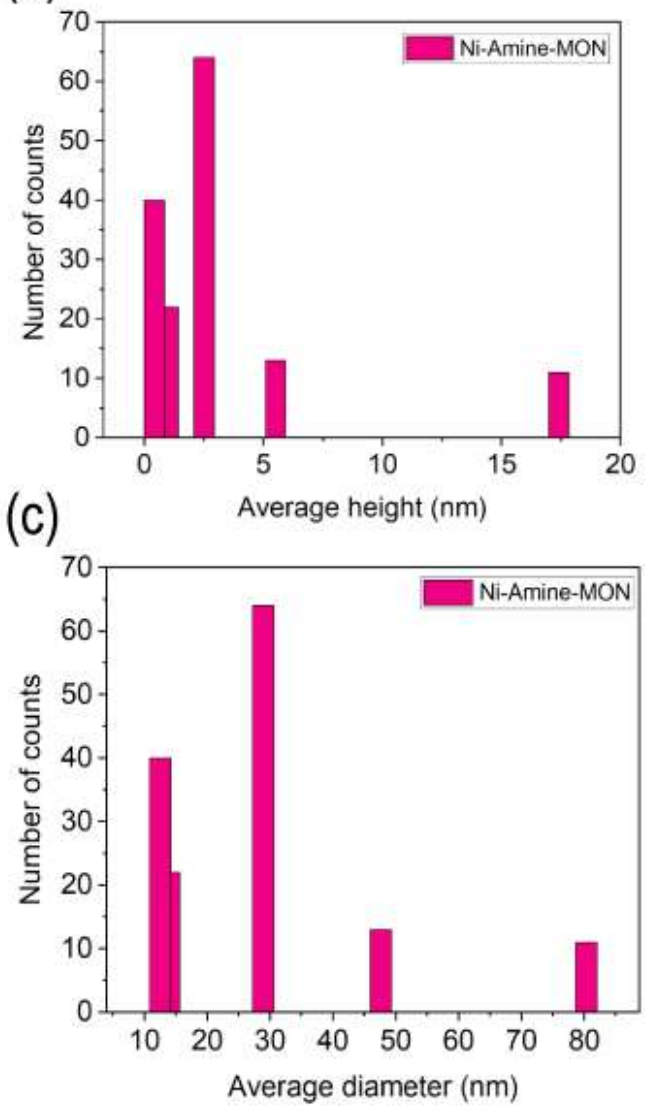

Figure S7. Average height and diameter of the Ni-Amine-MON characterized by AFM. (a) Imaging demonstrating the Ni-Amine-MON and the sample locations ( shown cyan colors) at which their average height and diameter are analyzed (b) Average height of the Ni-Amine-MON samples; the heights of the Ni-Amine-MONs were smaller of roughly 0.2 to $2 \mathrm{~nm}$ and few of the $\mathrm{Ni}$-Amine-MON samples were found to have height of $17 \mathrm{~nm}$. And the average diameter of the 
sample varies from $10 \mathrm{~nm}$ to $80 \mathrm{~nm}$. Y axis represents the number of counts at which the average values of height and diameters of Ni-Amine-MON are calculated.

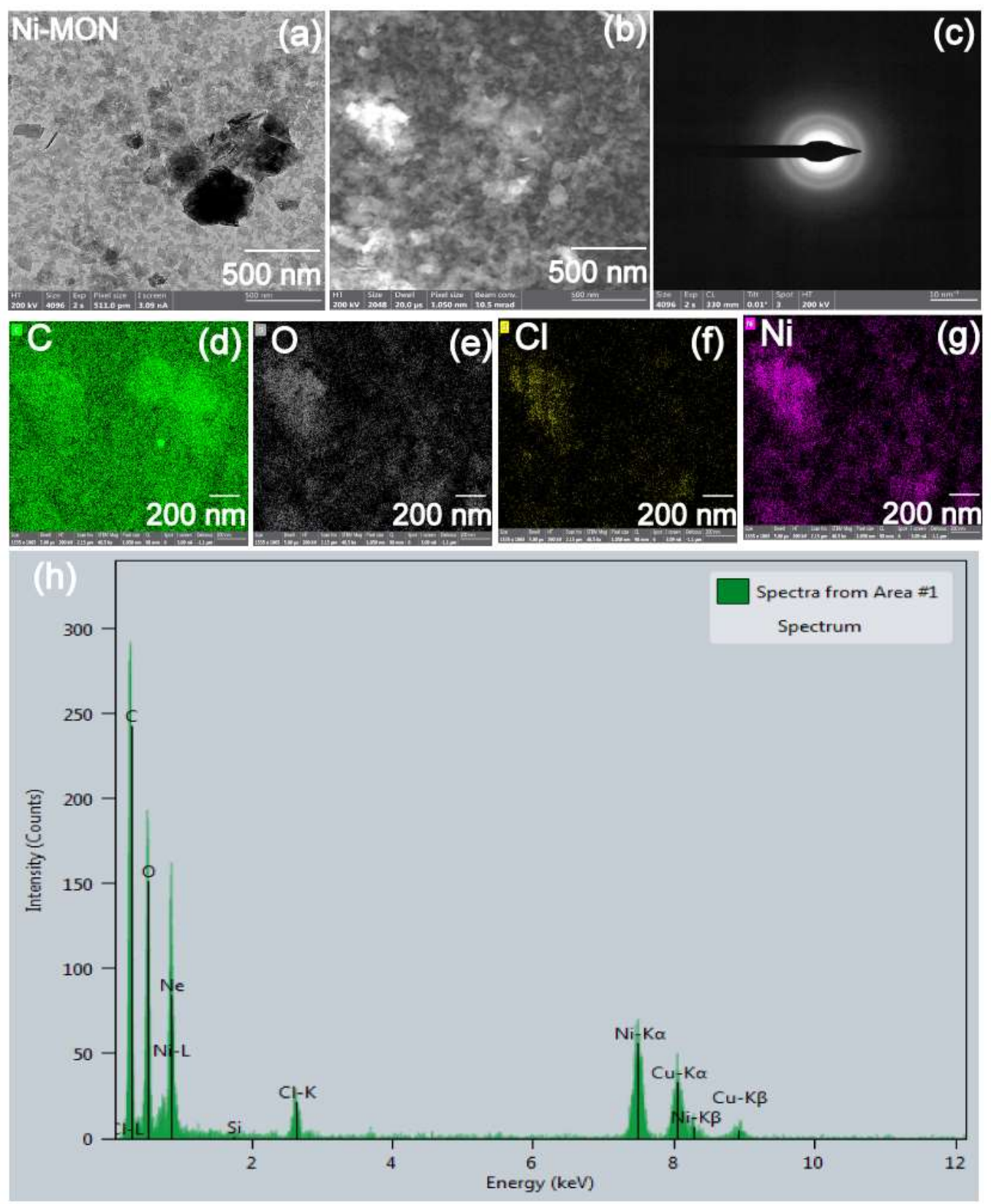

Figure S8. Structural information of Ni-MON (a) Transmission electron microscopic (TEM) image. Different size and shapes of Ni-MONs are shown. (b) HAADF image of Ni-MON (c) Xray diffraction pattern of Ni-MON; thin bright continuous circular pattern around the pointer attributing its slight crystal nature and slight amorphous form. (d-g) Elemental composition of the Ni-MON. (d) Carbon (e) Oxygen (f) Chlorine (g) Nickel. (h) Energy -dispersive spectra of $\mathrm{Ni}-\mathrm{MON}$ demonstrating the presence of elemental composition of $\mathrm{C}, \mathrm{O}, \mathrm{Cl}$, and $\mathrm{Ni}$. 

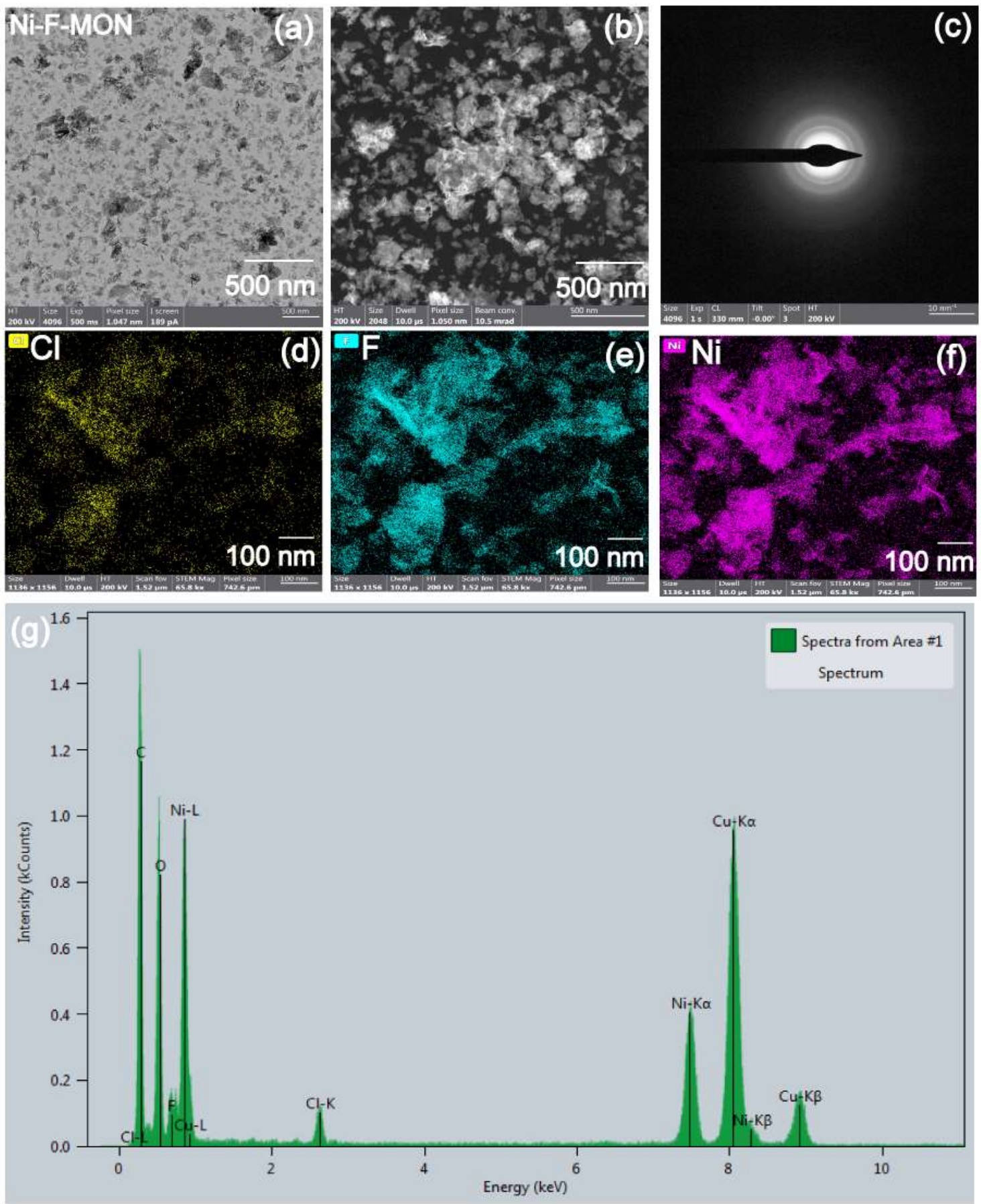

Figure S9. Structural topography of Ni-F-MON (a) TEM image; Different size and shapes of NiF-MONs; indicating slight porosity in the structure (b) HAADF image of Ni-F-MON (c) X-ray diffraction pattern of Ni-F-MON; thin bright continuous circular pattern around the pointer attributing its slight crystal nature and slight amorphous form. (d-f) Elemental composition of the Ni-F-MON. (d) Chlorine (e) Florine (f) Nickel (f) EDS spectra of Ni-F-MON demonstrating the presence of elemental composition of $\mathrm{Cl}, \mathrm{F}$, and $\mathrm{Ni}$. 

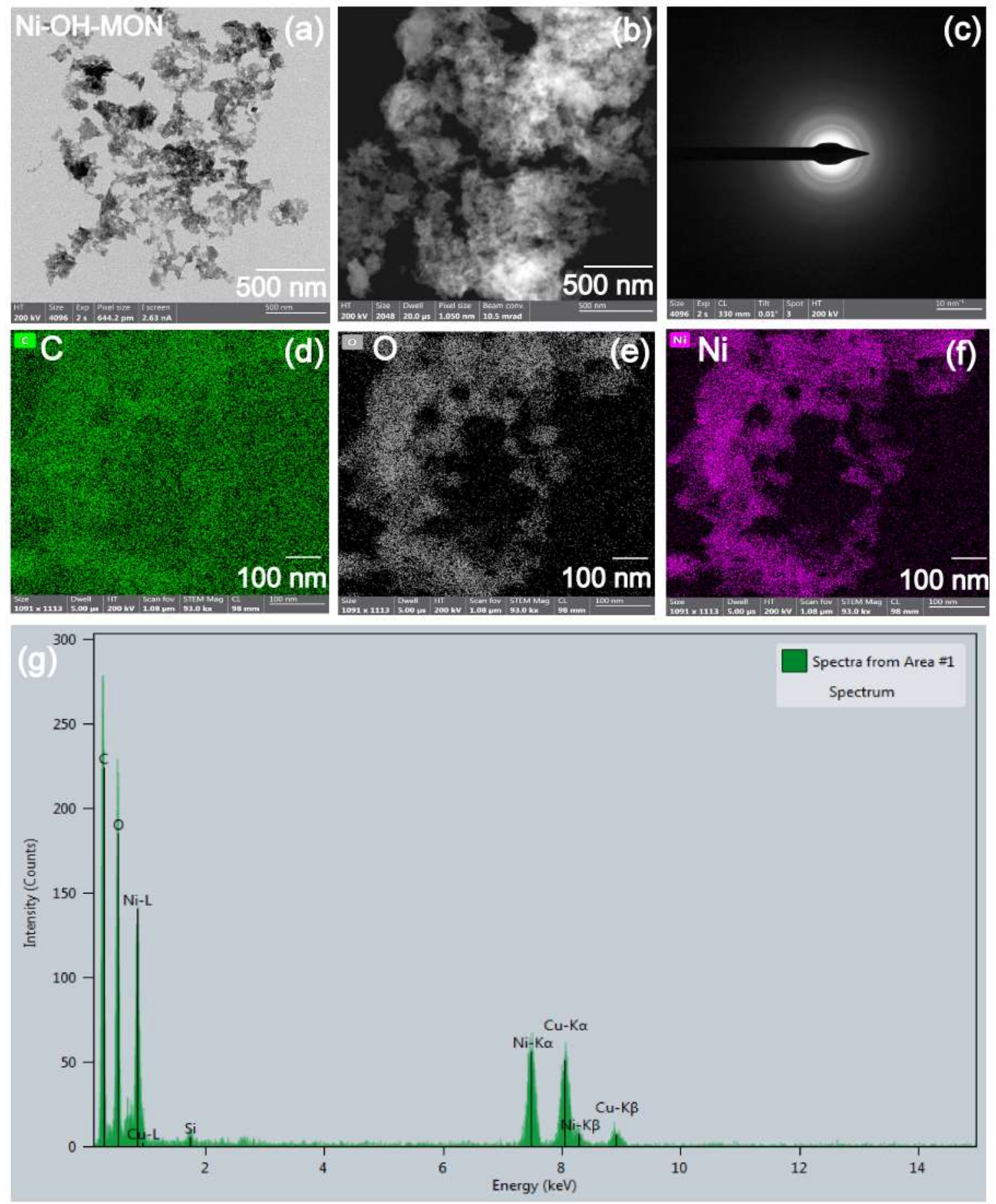

Figure S10. Structural topography of Ni-OH-MON (a) TEM image; Different size and shapes of Ni-OH-MONs; indicating slight porosity in the structure (b) HAADF image of Ni-F-MON (c) $\mathrm{X}$-ray diffraction pattern of Ni-OH-MON; thin bright continuous circular pattern around the pointer attributing its slight crystal nature and slight amorphous form. (d-f) Elemental composition of the Ni-OH-MON. (d) Carbon (e) Oxygen (f) Nickel (f) EDS spectra of Ni-OHMON demonstrating the presence of elemental composition of C, oxygen, and Ni. 

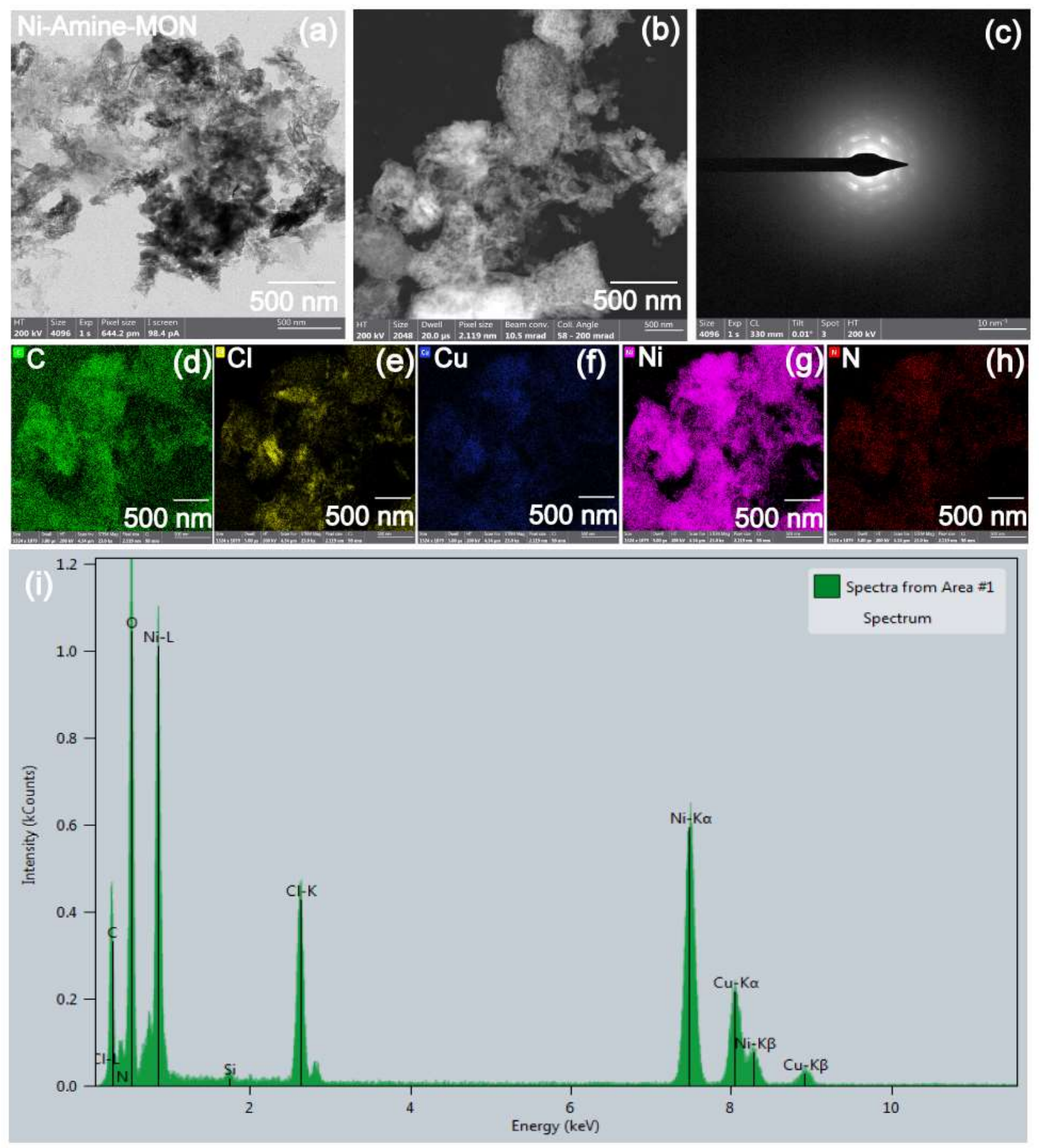

Figure S11. Structural topography of Ni-Amine-MON (a) TEM image; Different size and shapes of Ni-Amine-MONs; indicating slight porosity in the structure (b) HAADF image of Ni-AmineMON (c) X-ray diffraction pattern of Ni-Amine-MON; thin bright discontinuous circular pattern around the pointer attributing its more crystalline nature and slight amorphous form. (d-f) Elemental composition of the Ni-Amine-MON. (d) Carbon (e) Chlorine (f) Copper (g) Nickel (h) EDS spectra of Ni-Amine-MON demonstrating the presence of elemental composition of C, $\mathrm{Cl}, \mathrm{Cu}, \mathrm{Ni}, \mathrm{N}$. 


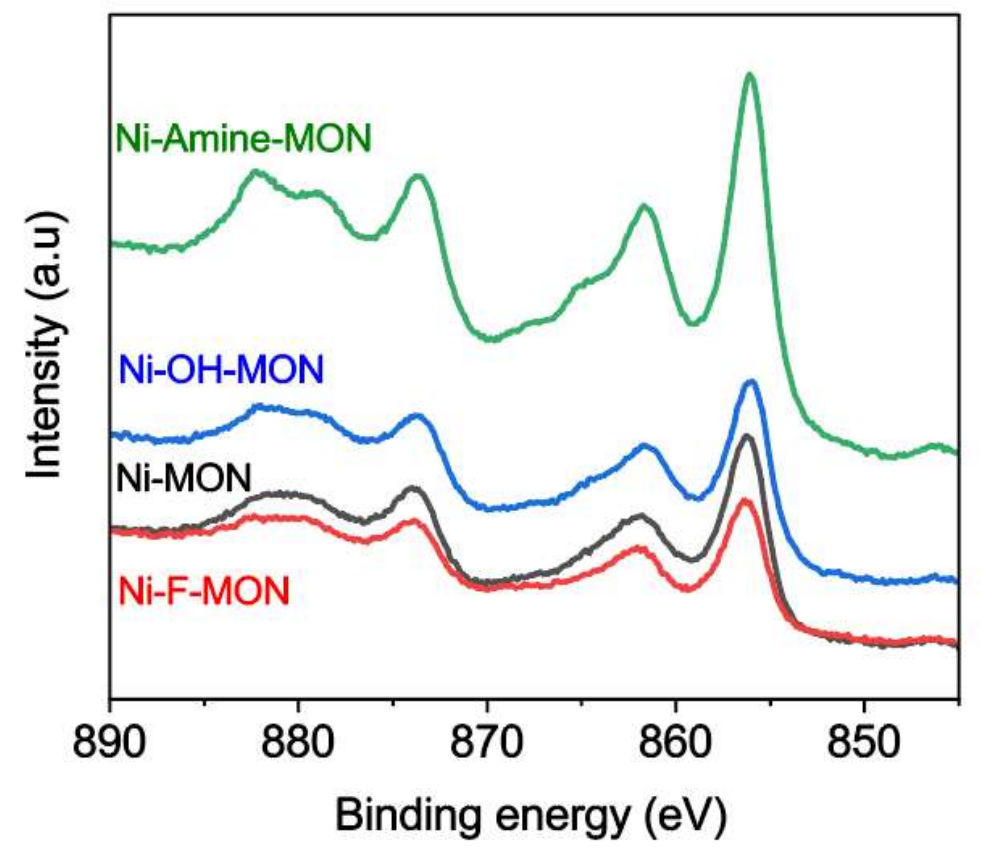

Figure S12. X-ray photoelectron spectroscopy of Ni-based MONs from 840- $890 \mathrm{eV}$. 

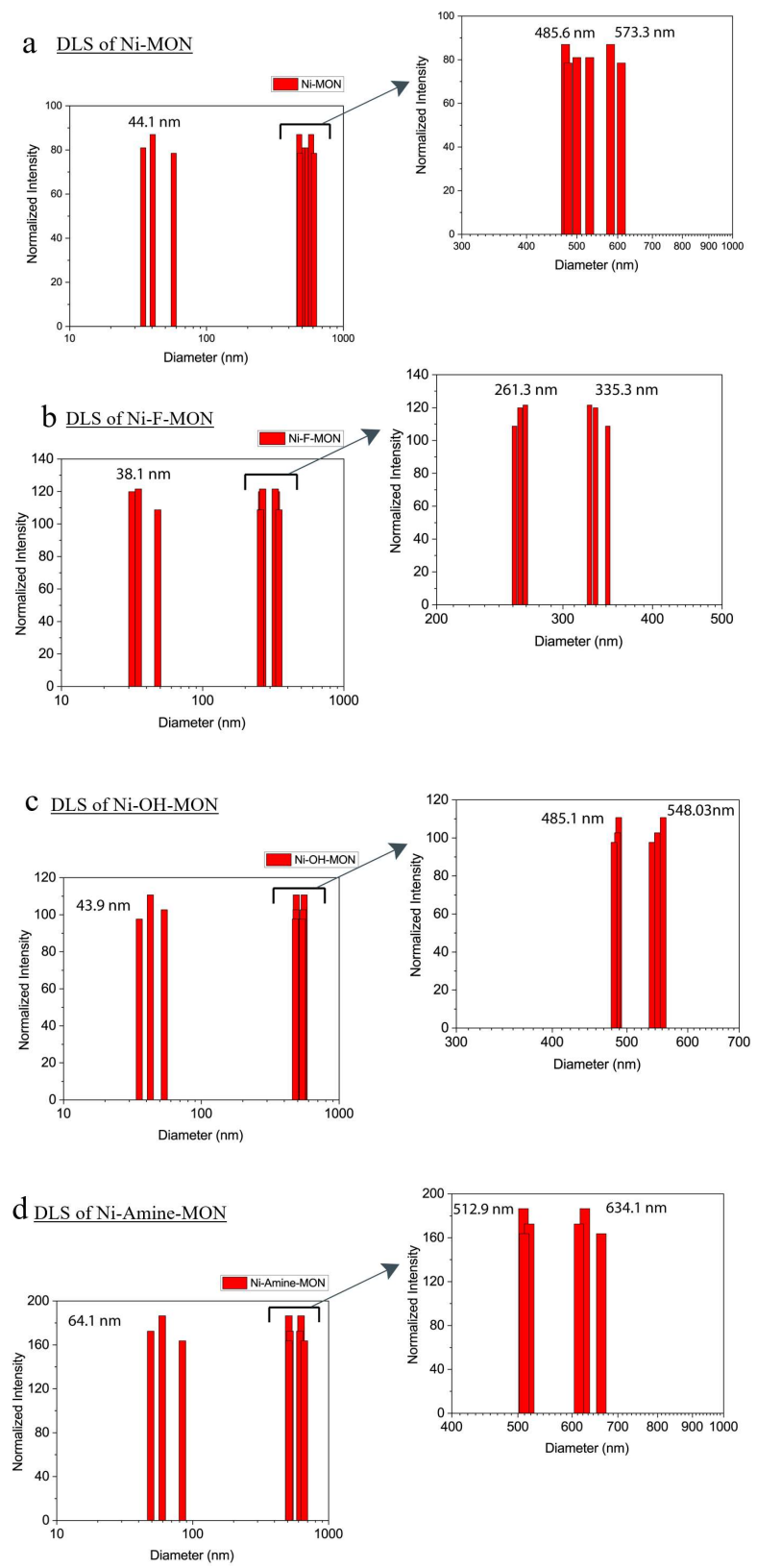

Figure S13: Dynamic light scattering (DLS) measurements performed on aqueous dispersions of the Ni-MON (a), Ni-F-MON (b), Ni-OH-MON (c), and Ni-Amine-MON (d). 

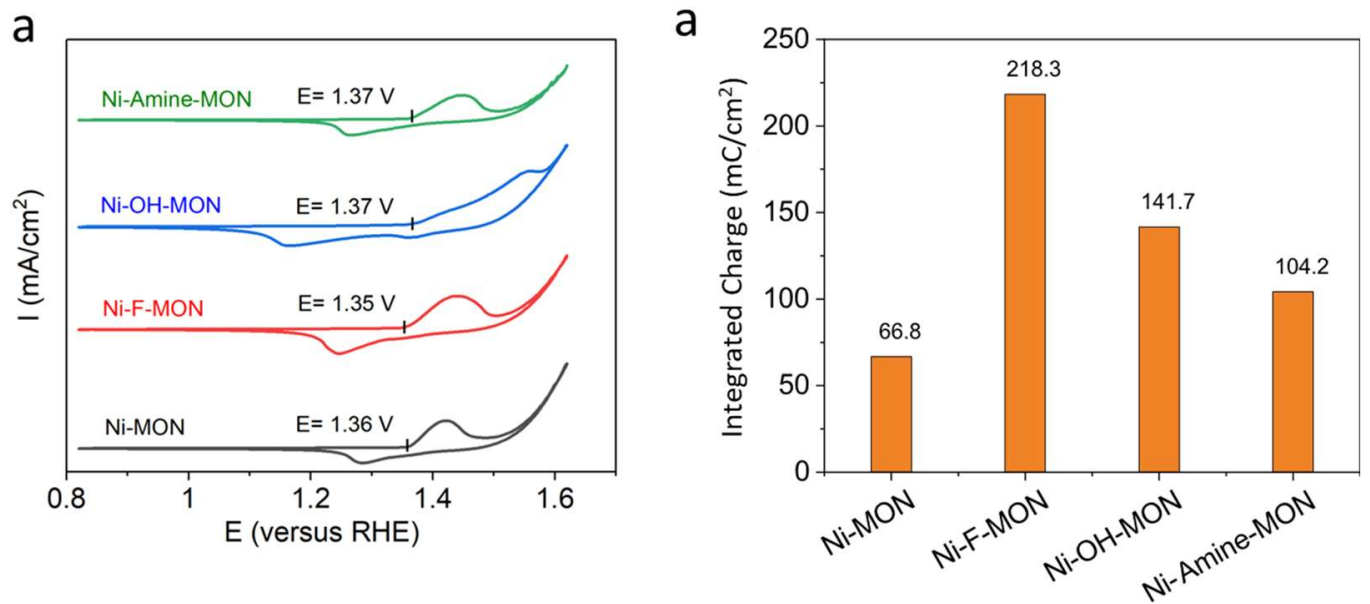

Figure S14: Cyclic voltammetry (CV) of Ni-based MONs. On a $1 \mathrm{~cm}^{2}$ area carbon paper electrode, the Catalyst ink of $200 \mu \mathrm{l}$ prepared by the Ni-based MONs were drop casted and allowed to dry completely. Then a $\mathrm{CV}$ of the MONs in $1 \mathrm{M} \mathrm{KOH}$ was performed at $5 \mathrm{mV} / \mathrm{s}$ (a). From this $\mathrm{CV}$, the charge attributed to the $\mathrm{Ni}(\mathrm{II} / \mathrm{III})$ redox was determined from integration of the reduction peak (b). 


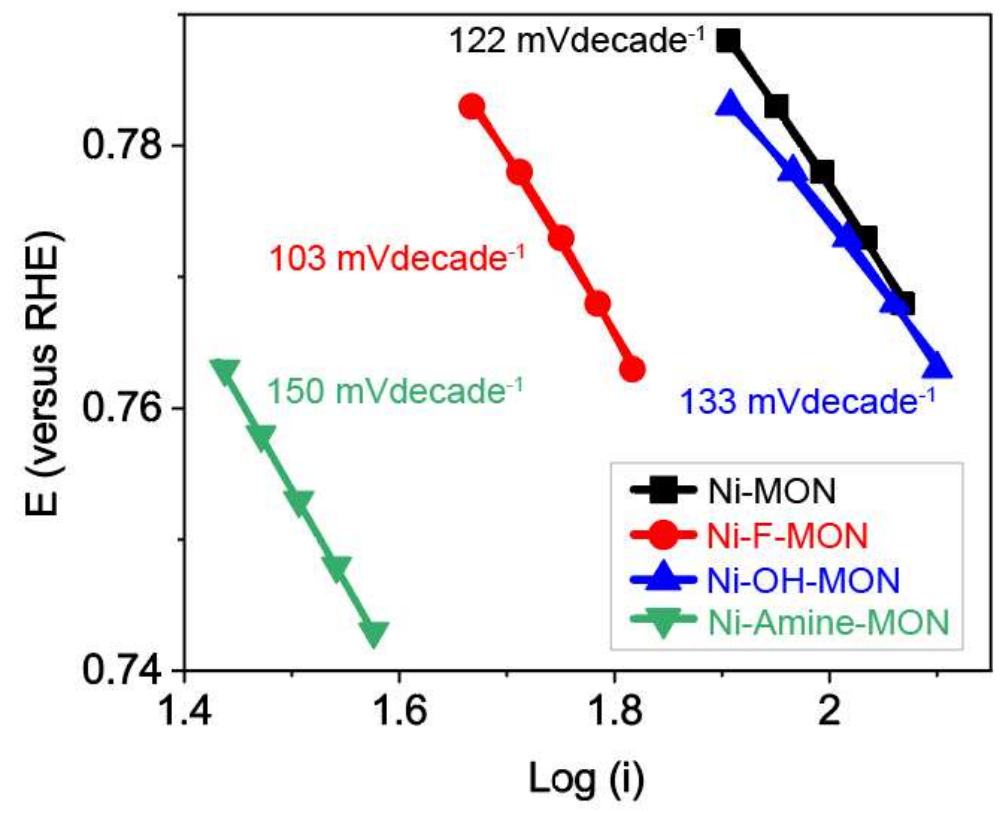

Figure S15: Tafel slopes derived from LSV measurements for each MON near the onset of the catalytic current. 


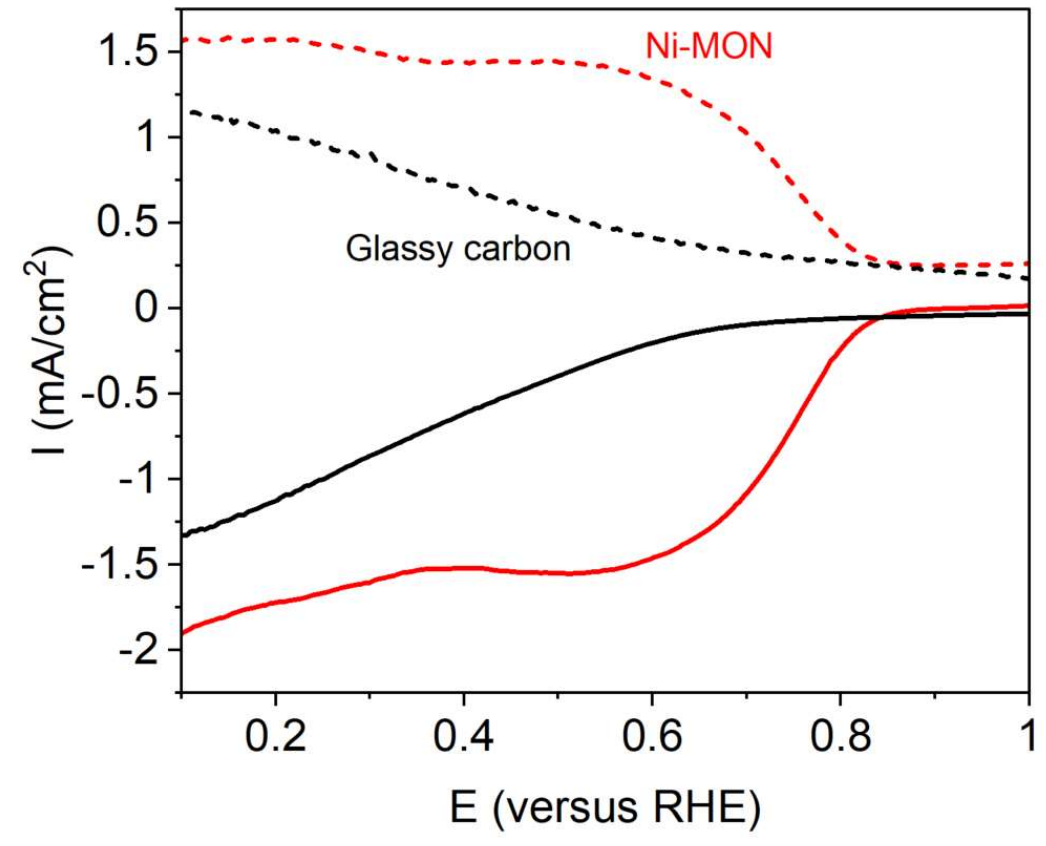

Figure S16: Linear sweep voltammetry of the bare glassy carbo, and the Ni-MON for comparison 


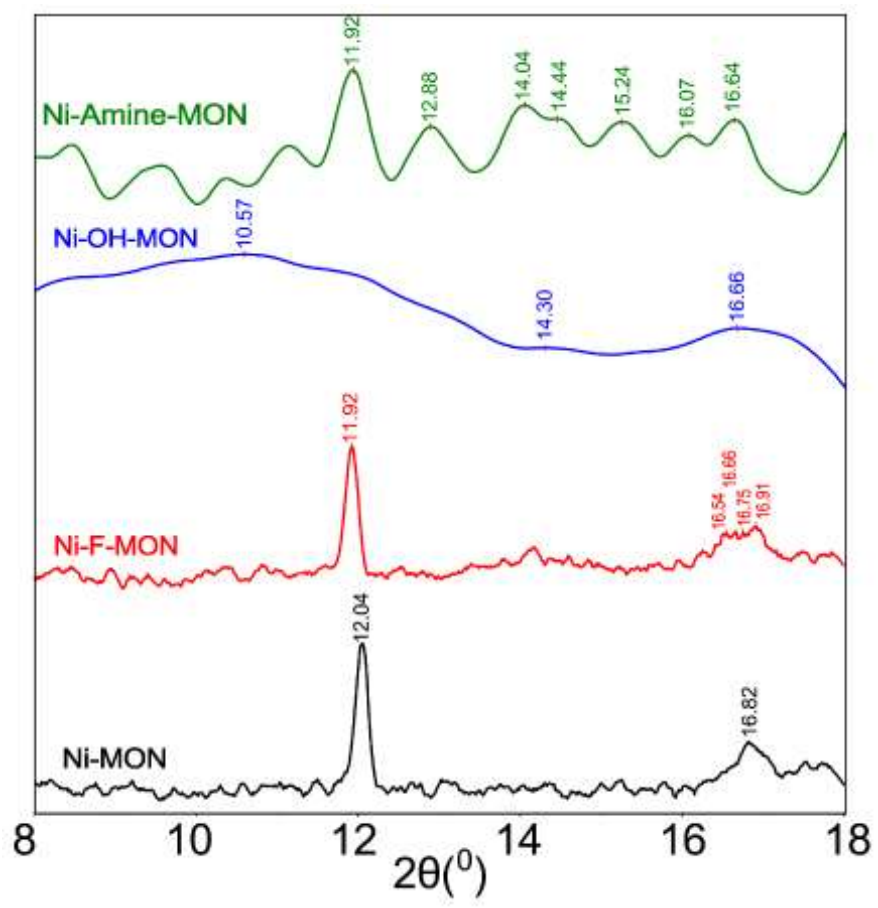

Figure S17. XRD of Ni catalyst ink deposited on carbon paper after one hour oxygen reduction reaction $\mathrm{Ni}-\mathrm{MON}$ at $-0.8 \mathrm{~V}$ RHE. 


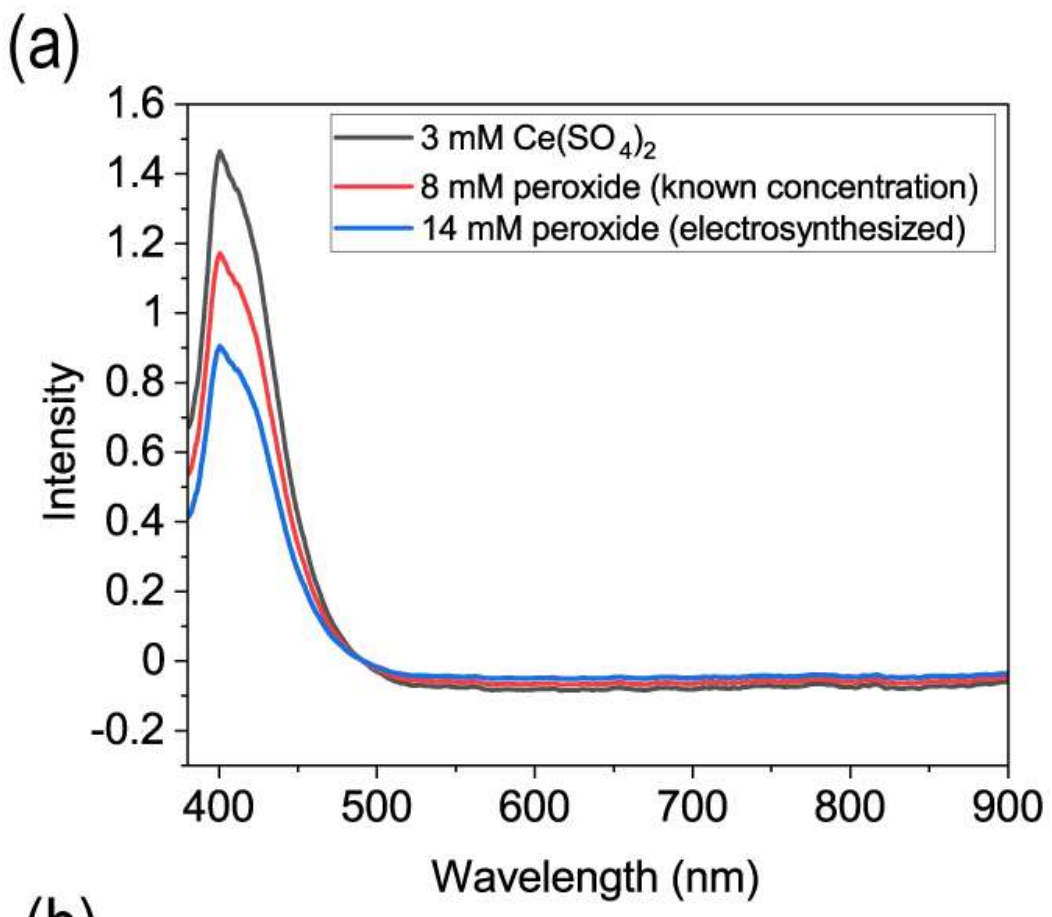

(b)

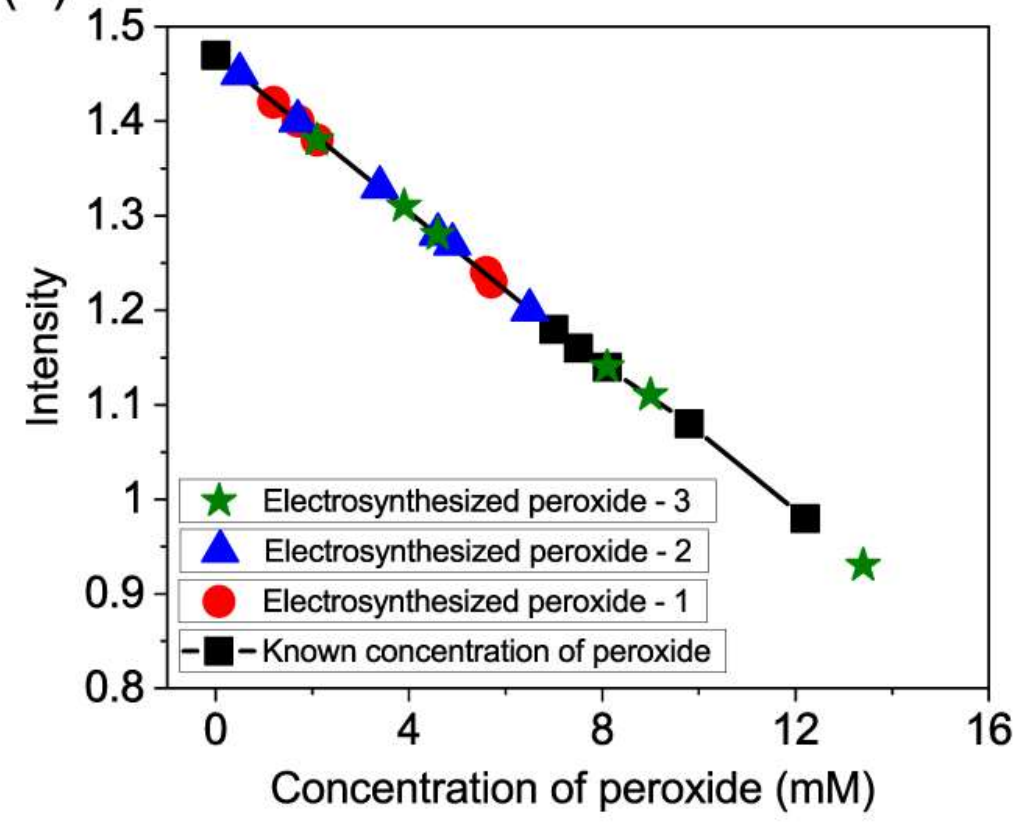

Figure S18. (a) UV-Vis absorption spectroscopy of synthesized peroxide titrating with the $\mathrm{Ce}\left(\mathrm{SO}_{4}\right)_{2}$. It is based on the mechanism that a yellow color solution of $\mathrm{Ce}^{4+}$ would be reduced by peroxide to colorless $\mathrm{Ce}^{3+}$. Representative spectra are shown in (a) and the calibration curve (absorption at $399.5 \mathrm{~nm}$ vs. peroxide concentration) alongside of several measurements is depicted in (b). 


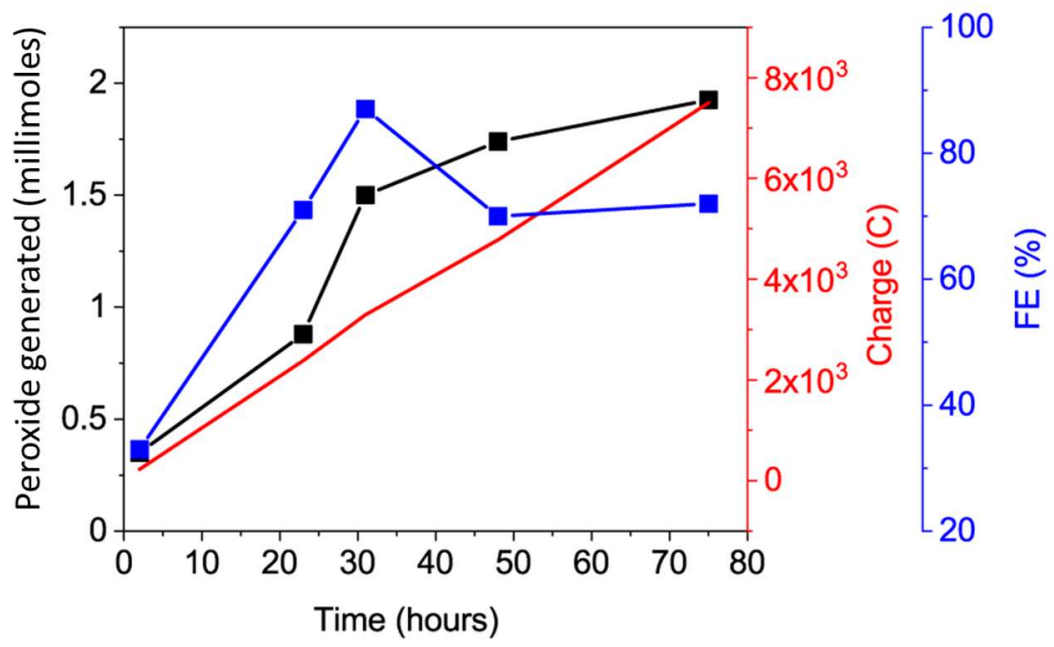

Figure S19: Long-term peroxide electrosynthesis with a Ni-Amine-MON deposited on a GDE. 